\title{
Mechanistically distinct cancer-associated mTOR activation clusters predict sensitivity to rapamycin
}

\author{
Jianing Xu, ${ }^{1}$ Can G. Pham, ${ }^{1}$ Steven K. Albanese, ${ }^{2,3}$ Yiyu Dong, ${ }^{1}$ Toshinao Oyama, ${ }^{1}$ Chung-Han Lee, ${ }^{1}$ Vanessa Rodrik-Outmezguine, ${ }^{4}$ \\ Zhan Yao, ${ }^{4}$ Song Han, ${ }^{1}$ David Chen, ${ }^{5}$ Daniel L. Parton, ${ }^{2}$ John D. Chodera, ${ }^{2,6}$ Neal Rosen, ${ }^{4,7}$ Emily H. Cheng, ${ }^{1,8,9}$ and James J. Hsieh ${ }^{1,7,10}$ \\ ${ }^{1}$ Human Oncology and Pathogenesis Program, ${ }^{2}$ Computational Biology Program, ${ }^{3}$ Gerstner Sloan Kettering Graduate School, and ${ }^{4}$ Molecular Pharmacology Program, Memorial Sloan Kettering Cancer Center, \\ New York, New York, USA. ${ }^{5}$ Oncology Global Development, Novartis Pharmaceuticals Corp., East Hanover, New Jersey, USA. ${ }^{6}$ Physiology, Biophysics, and Systems Biology Program, Weill Cornell Medical College, \\ Cornell University, New York, New York, USA. Department of Medicine and ${ }^{8}$ Department of Pathology, Memorial Sloan Kettering Cancer Center, New York, New York, USA. \\ ${ }^{9}$ Department of Pathology and Laboratory Medicine and ${ }^{10}$ Department of Medicine, Weill Medical College of Cornell University, New York, New York, USA
}

\begin{abstract}
Genomic studies have linked mTORC1 pathway-activating mutations with exceptional response to treatment with allosteric inhibitors of mTORC1 called rapalogs. Rapalogs are approved for selected cancer types, including kidney and breast cancers. Here, we used sequencing data from 22 human kidney cancer cases to identify the activating mechanisms conferred by mTOR mutations observed in human cancers and advance precision therapeutics. mTOR mutations that clustered in focal adhesion kinase targeting domain (FAT) and kinase domains enhanced mTORC1 kinase activity, decreased nutrient reliance, and increased cell size. We identified 3 distinct mechanisms of hyperactivation, including reduced binding to DEP domaincontaining MTOR-interacting protein (DEPTOR), resistance to regulatory associated protein of mTOR-mediated (RAPTORmediated) suppression, and altered kinase kinetics. Of the 28 mTOR double mutants, activating mutations could be divided into 6 complementation groups, resulting in synergistic Rag- and Ras homolog enriched in brain-independent (RHEBindependent) mTORC1 activation. mTOR mutants were resistant to DNA damage-inducible transcript 1-mediated (REDD1mediated) inhibition, confirming that activating mutations can bypass the negative feedback pathway formed between HIF1 and mTORC1 in the absence of von Hippel-Lindau (VHL) tumor suppressor expression. Moreover, VHL-deficient cells that expressed activating mTOR mutants grew tumors that were sensitive to rapamycin treatment. These data may explain the high incidence of mTOR mutations observed in clear cell kidney cancer, where VHL loss and HIF activation is pathognomonic. Our study provides mechanistic and therapeutic insights concerning mTOR mutations in human diseases.
\end{abstract}

\section{Introduction}

mTOR is a serine-threonine kinase that forms 2 distinct signaling complexes, mTORC1 and mTORC2 $(1,2)$, which are composed of common subunits (mTOR, mLST8, DEPTOR) $(3,4)$ and uniquely defined subunits. PRAS40 and regulatory associated protein of mTOR (RAPTOR) are specific to mTORC1 (5-10), whereas RICTOR, mSIN1, and PROTOR1/2 are specific to mTORC2 $(2,11-15)$. mTORC1 integrates nutrient and growth factor signaling to promote anabolic metabolism, such as protein synthesis and lipid synthesis (16-18), and to inhibit catabolic pathways, such as lysosome biogenesis and autophagy $(2,19-25)$. On the other hand, mTORC2 coordinates with PDK1 to phosphorylate/activate AKT, thereby regulating actin cytoskeleton, cell-cycle progression, and cellular survival (26).The efficacy of allosteric mTORC1 inhibitors everolimus and temsirolimus, 2 rapamycin analogs (rapalogs), in treating human cancers has been examined in clinical trials, leading to their approval by the US FDA for the treatment of kidney renal cell carcinoma (RCC) and estrogen receptor-positive (ER-positive) breast cancer (27-29). However, molecular mechanisms that underscore observed heterogeneous clinical benefits remain undetermined,

Conflict of interest: The authors have declared that no conflict of interest exists. Submitted: December 21, 2015; Accepted: June 2, 2016.

Reference information: J Clin Invest. 2016;126(9):3526-3540. doi:10.1172/JCI86120. and no predictive biomarkers are available to guide patient selection for therapy. Case studies of exceptional rapalog responders in kidney cancer, bladder cancer, and thyroid cancer demonstrated that loss-of-function mutations of tuberous sclerosis complex 1 (TSC1) and TSC2 or activating mutations of mTOR could be predictive for treatment response (30-34). On the other hand, a mutation in the FKBP-rapamycin-binding (FRB) domain of mTOR was reported to confer resistance to everolimus (33).

Clear cell RCC (ccRCC) is the most common ( $75 \%)$ type of human kidney cancer and is highly lethal when metastatic $(35,36)$. Genetically, ccRCC is characterized by biallelic inactivation of the von Hippel-Lindau (VHL) tumor suppressor gene, which encodes an E3 ubiquitin ligase that degrades HIF1 $\alpha$ and HIF2 $\alpha$ (37). Loss of VHL leads to uncontrolled accumulation of HIFs despite an adequately oxygenated tissue microenvironment, which in turn results in constitutive activation of HIF-target genes that regulate angiogenesis, resulting in the known highly vascular nature of ccRCC (38). Accordingly, it was postulated that the universal, early loss of VHL would render ccRCC susceptible to the inhibition of the VEGF signaling pathway (39). Indeed, the mainstream treatment for metastatic ccRCC encompasses antibody against VEGF or inhibitors of VEGFR, such as bevacizumab $(40,41)$, sorafenib $(42,43)$, sunitinib $(44,45)$, pazopanib $(46,47)$, and axitinib $(48$, 49). However, the complete functional loss of VHL alone was 
A

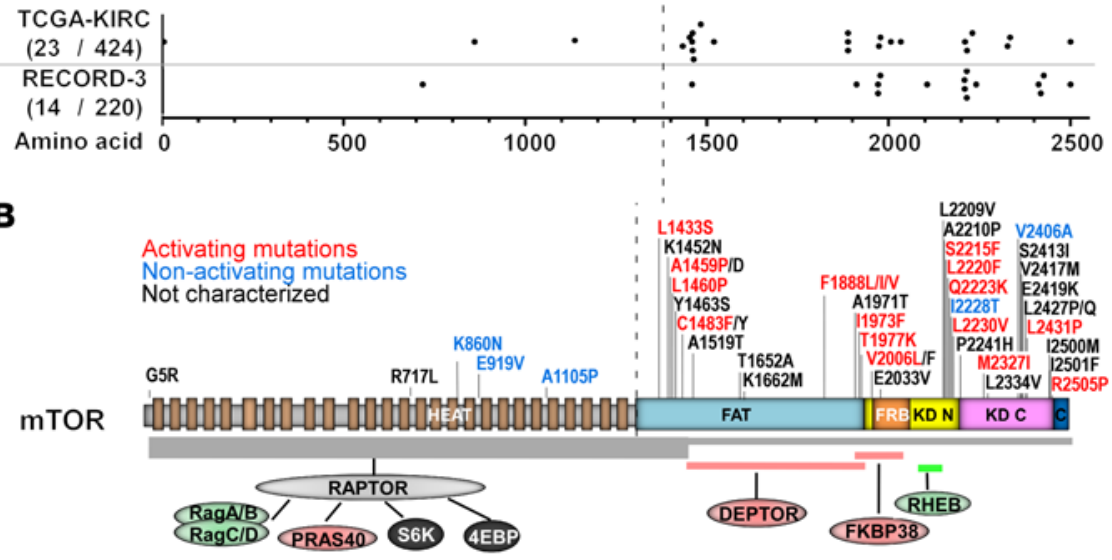

C
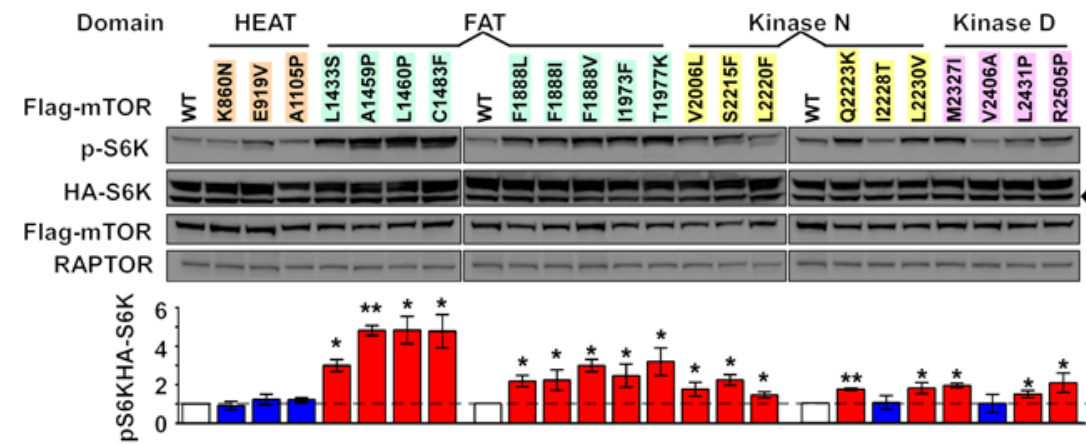

D

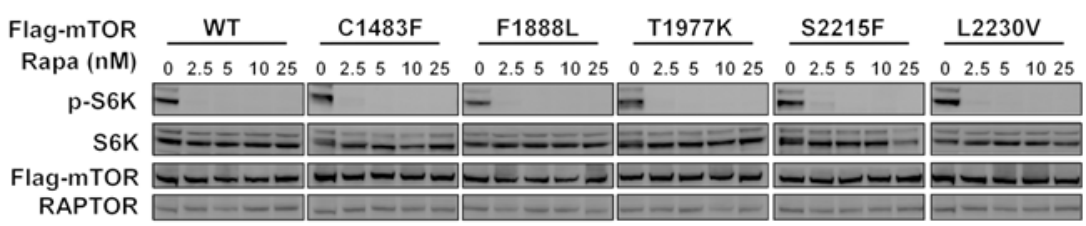

Figure 1. Characterization of kidney cancer-derived mTOR missense mutations. (A) Amino acid positions of mTOR missense mutations identified in ccRCC (TCGA_KIRC and RECORD-3). Numbers in parentheses indicate number of patients with $\mathrm{MTOR}$ missense mutations versus total number of patients sequenced ( 2 patients in the RECORD-3 study carry 2 different mTOR mutations). (B) Diagram shows the domain structure of mTOR, its regulatory interaction partners (negative regulators in pink, positive regulators in green, and a dual-role regulator in gray), and the substrates of mTORC1 complex. The positions within mTOR that are involved in the interaction with the regulatory partners are highlighted below the domain structure. The thickness of the horizontal bar of RAPTOR-mTOR interaction indicates the relative binding affinity. mTOR missense mutations derived from ccRCC are mapped and color coded to summarize their respective effects on mTORC1 signaling (activating mutations in red). KD N, kinase domain $\mathrm{N}$ lobe; $\mathrm{KD}$ C, kinase domain $\mathrm{C}$ lobe. (C) Most of the mTOR mutants in the FAT and kinase domains induce higher levels of S6K phosphorylation (T389) than WT mTOR. 293T cells were transfected with vectors expressing HA-tagged S6K and Flag-tagged mTOR. Forty-eight hours later, cells were lysed and whole cell lysates were subjected to immunoblot analysis using the indicated antibodies. Densitometry of phosphorylated S6K versus HA-S6K from 3 independent experiments is shown (mean \pm SEM, $n=3$ independent experiments). ${ }^{*} P<0.1 ;{ }^{* *} P<0.05$ ( $t$ test). The arrowhead denotes a cross-reactive band. (D) All mTOR mutants tested are sensitive to rapamycin treatment. Tetracycline-inducible HeLa cells expressing WT or mutant mTOR were treated with the indicated doses of rapamycin for 1 hour prior to immunoblot analysis using the indicated antibodies. insufficient to cause ccRCC in various mouse models, indicating the requirement of additional genetic/epigenetic events (50-53). One candidate pathway is the mTOR signaling pathway. Evidence supporting such a scenario includes the following: (a) inhibitors of mTORC1 everolimus and temsirolimus are 2 standard treatment options for patients with metastatic RCC (mRCC), and (b) clustered, recurrent missense mutations of $\mathrm{mTOR}$ were observed in approximately $5 \%$ of cases of ccRCC (54-58). Hence, we envisioned that studying human kidney cancer-derived mTOR missense mutations could elucidate regulatory mechanisms of mTOR in cancers and other human diseases carrying mTOR mutations and affect treatment decisions.

Based on available human cancer genomic databases, there are approximately 570 missense mutations of mTOR identified across 20 cancer types that occur at various frequencies and at different amino acid positions (Supplemental Figure 1 and Supplemental Table 1; supplemental material available online with this article; doi:10.1172/JCI86120DS1). These mutations present a challenge, but could also offer an opportunity to enroll cancer patients into histology-independent, genomics-guided, mutation-enriched "basket" trials (59). To implement this potential therapeutic strategy, an imperative step is to functionally interrogate cancer-derived mTOR missense mutations in a systemic fashion. Several regula- tory mechanisms concerning the activation of WT mTORC1 have been proposed, which involves mTOR-interacting proteins RAG, Ras homolog enriched in brain (RHEB), DEP domain containing MTOR-interacting protein (DEPTOR), RAPTOR, PRAS40, and FKBP38 (2, 4-10, 19, 60-63). Indeed, a recent study surveyed cancer-derived mTOR activation mutations, which exploited the DEPTOR-centered mechanism (64). However, how activating mutations contribute to the pathogenesis of cancer, especially kidney cancer, and what molecular mechanisms underlie individual activating mutations beyond DEPTOR remain unknown.

\section{Results}

Kidney cancer-derived mTOR missense mutations clustered at FAT and kinase domains are activating mutations. The Cancer Genome Atlas Kidney Renal Clear Cell Carcinoma (TCGA-KIRC) project, consisting of stage I-IV cases (stages I-II, 257 cases [57.6\%]; stages III-IV, 189 cases [42.4\%]), has identified mTOR missense mutations at 5.4\% (54). The RECORD-3 trial (Renal Cell Cancer Treatment with $\underline{\text { Oral }}$ RAD001 Everolimus Given Daily) (65), consisting of all stage IV cases (stage IV, 258 cases sequenced [100\%]), identified mTOR missense mutations at $6 \%(P=0.67, t$ test, comparison between the mutation rates between 2 studies) (Figure $1 \mathrm{~A}$ and Supplemental Table 1) $(65,66)$. Together, these findings support 
A

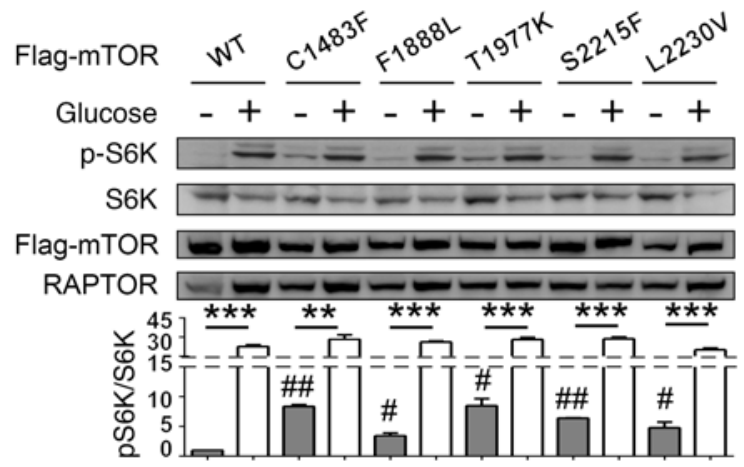

C

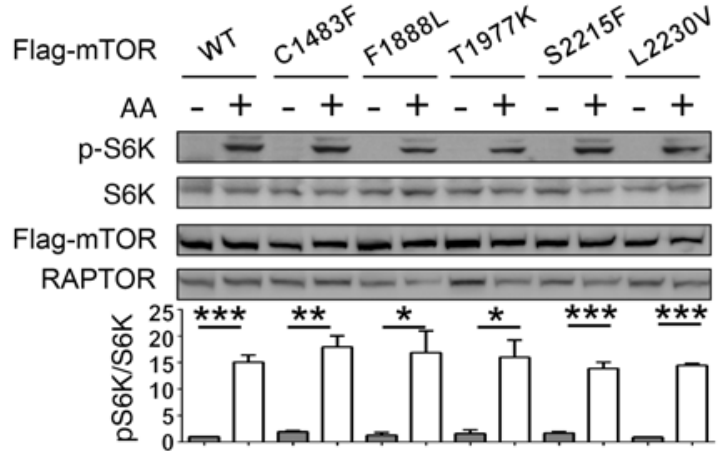

B

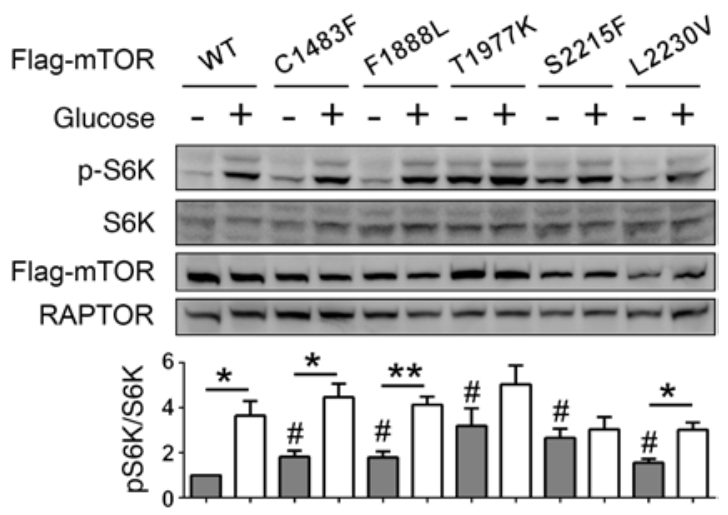

D

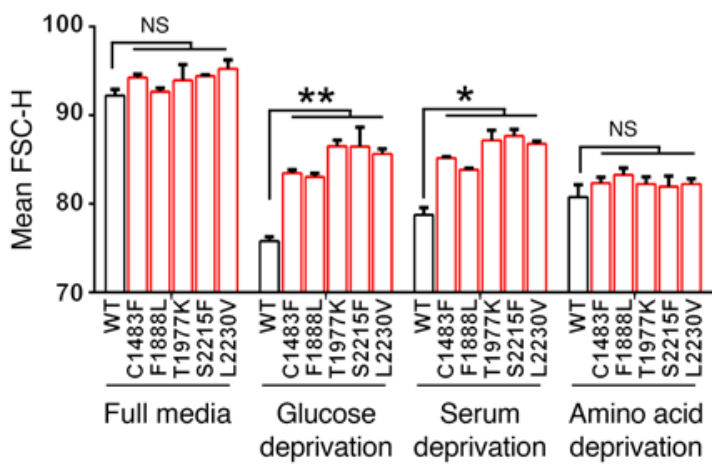

Figure 2. Characterization of nutrient dependence of mTORC1 signaling conferred by mTOR-activating mutations. (A and B) Tetracycline-inducible HeLa cells expressing WT or mutant mTOR were either starved for glucose (A) or serum (B) for 1 hour or starved for 1 hour and restimulated with full media for 1 hour and subsequently subjected to immunoblot analysis. (C) Tetracycline-inducible HeLa cells expressing WT or mutant mTOR were either starved for amino acid for 1 hour or starved for 1 hour and restimulated with amino acid for 1 hour. Whole cell lysates were subjected to immunoblot analysis using the indicated antibodies. In A-C, densitometry of phosphorylated S6K versus HA-S6K from 3 independent experiments is shown (mean $\pm \mathrm{SEM}, n=3$ independent experiments). ${ }^{*} P<0.05 ;{ }^{* *} P<0.01 ;{ }^{* *} P<0.001$ ( $t$ test). ${ }^{*} P<0.05 ;{ }^{* \#} P<0.01$ ( $t$ test, comparison between indicated mutants and WT). (D) Tetracycline-inducible HeLa cells expressing WT or mutant mTOR under the indicated culture conditions were subjected to flow cytometry analysis for cell size. Data shown are the mean forward scatter height (FSC-H) from 3 independent experiments. Error bars represent SEM. ${ }^{*} P<0.05 ;{ }^{* *} P<0.01$ ( $t$ test).

the idea that hyperactive $\mathrm{mTORC1}$ resulting from mTOR-activating mutations could constitute an oncogenic driver event in kidney cancer pathology (53).

Although mTOR missense mutations scattered through the protein in most cancers, they clustered within the focal adhesion kinase targeting domain (FAT) and kinase domains in kidney cancer from these 2 independent studies (Figure 1, A and B). To investigate the functional consequence, we generated 22 individual missense mTOR mutants and examined their impact on mTORC1 activity by assessing the phosphorylation of S6K and 4EBP1. When individual mTOR mutants were coexpressed with HA-S6K or HA-4EBP in 293T cells, the majority of FAT and kinase domain mutants exhibited higher activity, whereas HEAT domain mutants exhibited similar mTORC1 kinase activity with respect to WT mTOR (Figure 1C and Supplemental Figure 2). Meanwhile, when individual mutants were coexpressed with HA-AKT1, most mutants exhibited activity similar to that of WT mTOR (Supplemental Figure 3). Therefore, in this study we denote mTOR mutations exhibiting significantly higher mTORC1 activity than WT mTOR as activating mutations and those with similar activity as nonactivating mutations. Notably, a wider range of activities toward S6K than 4EBP based on phosphorylation was observed among mTOR-activating mutations, which might be due to differential "substrate quality" between S6K and 4EBP (67). These results suggest that $\mathrm{S} 6 \mathrm{~K}$ could discern differences of mTORC1 activity better than 4EBP. Hence, we mainly utilized phosphorylated $\mathrm{S} 6 \mathrm{~K}$ as the readout of mTORC1 activity for this study. Similar results on S6K and AKT phosphorylation were obtained when individual mTOR constructs were expressed at a level comparable to that of endogenous mTOR protein using an mTOR-silenced 293T cell line (Supplemental Figures 4 and 5). To determine the sensitivity of these hyperactive mTOR mutants to rapamycin, we generated $6 \mathrm{HeLa}$ cell lines expressing tetracycline-inducible WT or mutant mTOR that corresponds to different mutation clusters (Supplemental Figure 6). Notably, all these hyperactive mTOR mutants remained sensitive to rapamycin (Figure 1D), which is consistent with prior reports $(30,31,64)$.

mTOR-activating mutants are more resistant to glucose and serum but not to amino acid deprivation than WT MTOR. Diverse nutrient and growth factor signals converge on small GTPases RAG and RHEB to coordinate mTORC1 activation $(2,19)$. Amino acids activate $\mathrm{RAGA} / \mathrm{B}^{\mathrm{GTP}} ; \mathrm{C} / \mathrm{D}^{\mathrm{GDP}}$, which recruits mTORC1 
A

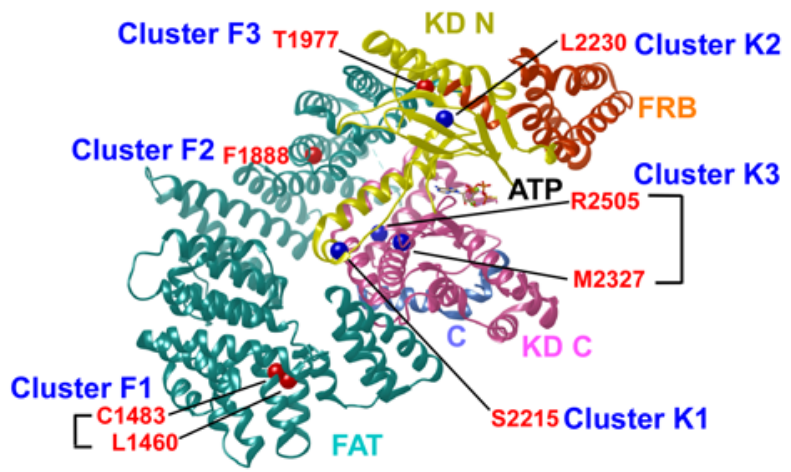

B

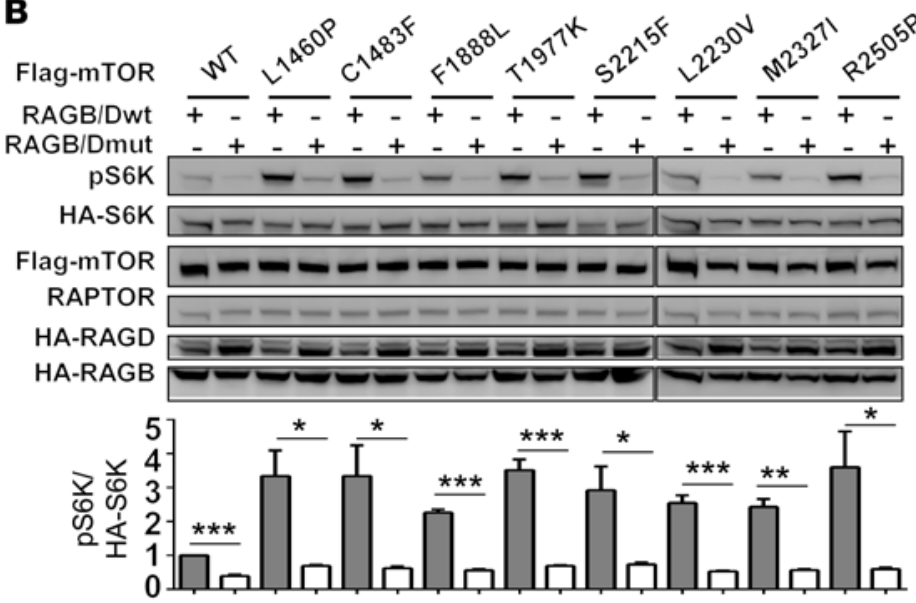

C
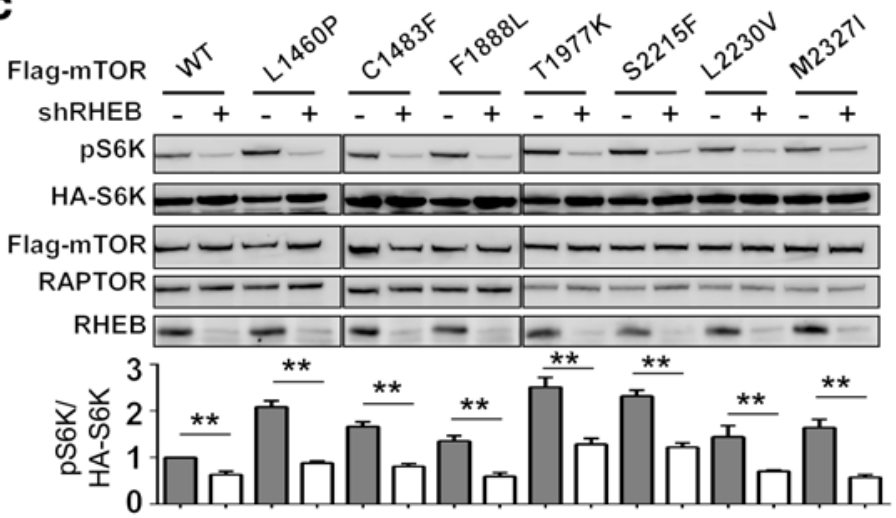

to the lysosome $(2,19,60,61)$. Growth factor and glucose signals inactivate the TSC (68-75), which results in the lysosomal accumulation of RHEB ${ }^{\mathrm{GTP}}$ that directly activates mTORC1 (2, 19, 76-78). To determine whether activating mTOR mutations affect the sensitivity of mTORC1 signaling to nutrient deprivation, we used the aforementioned tetracycline-inducible mTOR-activating mutant HeLa cells (Supplemental Figure 6). Although all of the mTOR mutants were equal to WT mTOR in sensitivity to amino acid deprivation based on S6K phosphorylation, they displayed varying resistance to glucose or serum deprivation (Figure 2, A-C and Supplemental Figure 7, A-C). Given that increased cell size is one of the best-characterized physiological readouts of mTORC1 activation (79), we assessed the effects of individual mTOR mutations on cell size under full media or nutrient-deprived conditions using flow cytometry. Although HeLa cells expressing WT or mutant mTOR were
Figure 3. Characterization of the dependency of mTOR-activating mutants on RAG and RHEB. (A) Structural model of FAT, FRB, and kinase domains of mTOR. Mutation clusters and selected mutations in each cluster are indicated. (B) 293T cells were transfected with vectors expressing HA-S6K, Flag-mTOR, and either RAGB plus RAGD (RAGB/D ${ }_{\text {WT }}$ ) or dominant negative RAGB ${ }^{\text {GDP }}$ plus $R A C D^{G T P}\left(R A G B / D_{\text {Mut }}\right.$ ). Whole cell lysates were subjected to immunoblot analysis using the indicated antibodies. (C) HeLa cells stably expressing shRNA against GFP or RHEB were transfected with vectors expressing HA-S6K and the indicated Flag-mTOR mutants and subjected to immunoblot analysis using the indicated antibodies. In B and C, densitometry of phosphorylated S6K versus $\mathrm{HA}-\mathrm{S} 6 \mathrm{~K}$ from 3 independent experiments is shown (mean \pm SEM, $n=3$ independent experiments). ${ }^{*} P<0.05 ;{ }^{*} P<0.01$; ${ }^{* * *} P<0.001$ ( $t$ test).

comparable in size under full media, mTOR-mutant HeLa cells were larger than mTOR-WT HeLa cells upon glucose or serum deprivation (Figure 2D). Consistent with the complete dependence of mutant mTORC1 activity on amino acid as WT mTORC1 (Figure 2C and Supplemental Figure 7C), mTOR mutant HeLa cells were comparable in size to mTOR-WT HeLa cells upon amino acid deprivation (Figure 2D). Collectively, our data demonstrated that kidney cancer-derived mTOR-activating mutations appear to sustain mTORC1 kinase activity under glucose- or serum-limited but not amino acid-limited conditions.

All cancer-derived $m$ TOR-activating mutants require $R A G$ and RHEB for activation. The crystal structure of mTOR was recently reported, which reveals that the FAT domain forms a $\mathrm{C}$-shaped solenoid that wraps around and clamps the V-shaped kinase domain (Figure 3A) (80). This level of structural resolution enabled us to accurately position individual mTOR mutations and allow for structure-based assignment of 6 distinct clusters, including 3 in the FAT domain (F1, F2, F3) and the other 3 in the kinase domain (K1, K2, K3) (Figure 3A). Several regulatory mechanisms concerning the activation of WT mTORC1 have been proposed, including mTOR-interacting proteins RAG, RHEB, DEPTOR, RAPTOR, PRAS40, and FKBP38 (2, 4-10, 19, 60-63) (Figure 1B). We hypothesized that distinct mTOR mutation clusters might affect the interaction/regulation between mTOR and specific mTOR regulators, contributing to mTORC1 activation.

The activation of mTORC1 requires RAG and RHEB, which mediate lysosome localization and direct activation, respectively $(2,76)$. Accordingly, we tested to determine whether overexpression of dominant negative $\mathrm{RAGB}^{\mathrm{GDP}} / \mathrm{RAGD}^{\mathrm{GTP}}$ (61) or knockdown of RHEB affected the activity of mTOR mutants in phosphorylating $\mathrm{S} 6 \mathrm{~K}$. All the activating mTOR mutants were sensitive to $\mathrm{RAGB}^{\mathrm{GDP}} / \mathrm{RAGD}^{\mathrm{GTP}}$ (Figure $3 \mathrm{~B}$ ), which is consistent with the fact that these mTOR mutants were sensitive to amino acid deprivation (Figure 2, C and D). Furthermore, although T1977K and S2215F show slightly higher residual activities upon RHEB knockdown, all these mutants still required RHEB for activation (Figure 3C). In summary, cancer-derived mTORactivating mutations from all 6 clusters remain dependent on RAG and RHEB for mTORC1 signaling. 
A

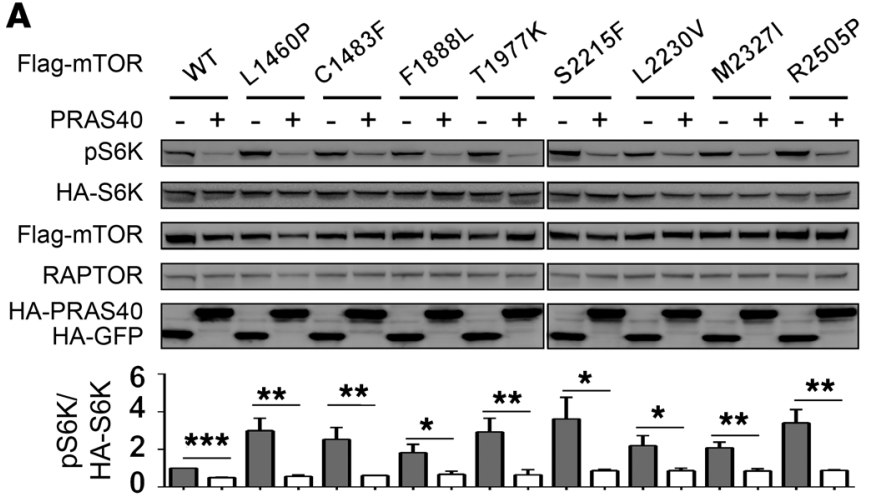

B

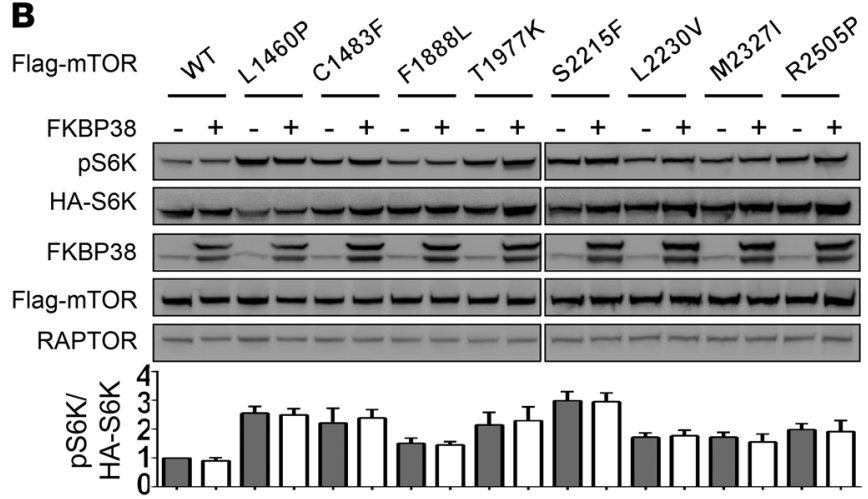

D

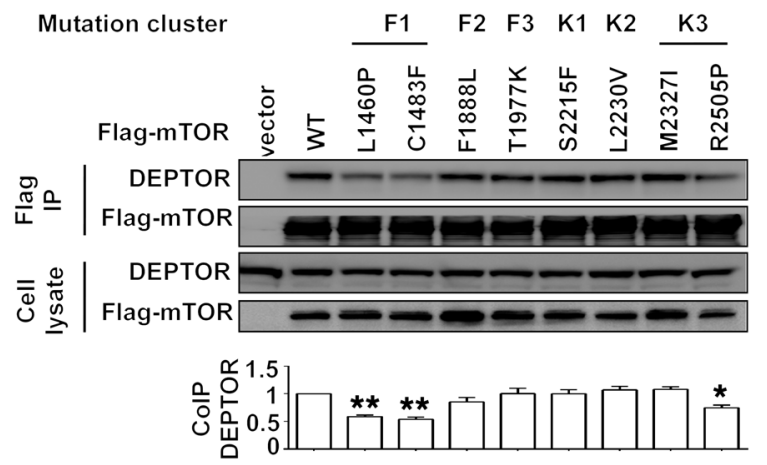

Figure 4. Characterization of the response of mTOR-activating mutants to negative regulators PRAS40, FKBP38, and DEPTOR. (A) Inhibition of mTOR-activating mutants by PRAS40. 293T cells were transfected with vectors expressing HA-S6K, Flag-mTOR, and either GFP or PRAS40 and subjected to immunoblot analysis using the indicated antibodies. (B) 293T cells were transfected with vectors expressing HA-tagged S6K, Flag-tagged WT, or mutant mTOR, and either GFP or FKBP38. Whole cell lysates were subjected to immunoblot analysis using the indicated antibodies. (C) Tetracycline-inducible 293T cells expressing shRNA against FKBP38 were transfected with vectors expressing HA-S6K and the indicated Flag-mTOR mutants, followed by either induction of FKBP38 with doxycycline or not. Whole cell lysates were subjected to immunoblot analysis using the indicated antibodies. In A-C, densitometry of phosphorylated S6K versus HA-S6K from 3 independent experiments is shown (mean \pm SEM, $n=3$ independent experiments). ${ }^{*} P<0.1 ;{ }^{* *} P<0.05$; ${ }^{* * *} P<0.01$ ( $t$ test). (D) $293 \mathrm{~T}$ cells, transfected with a control vector or vectors expressing the indicated WT or mutant Flag-mTOR were subjected to anti-Flag immunoprecipitation. The input $(10 \%)$ and immunoprecipitates were analyzed by the indicated immunoblots. Densitometry of DEPTOR present in anti-Flag immunoprecipitates is shown (mean $\pm \mathrm{SEM}, n=3$ independent experiments). ${ }^{*} P<0.1 ;{ }^{* *} P<0.05$ ( $t$ test, comparison between indicated mutants and WT).

All mTOR-activating mutants are sensitive to negative regulator PRAS40, yet display differential binding to negative regulator DEP$T O R$. We next examined the effect of 3 reported negative regulators of mTORC1, PRAS40, FKBP38, and DEPTOR, on activating mTOR mutants. Overexpression of PRAS40 inhibited all the tested mTOR mutants from phosphorylating S6K (Figure 4A), which is consistent with the known mechanism of PRAS40 that occurs through disrupting RAPTOR-mediated mTORC1 substrate recognition, and the necessity of RAPTOR in mTORC1 signaling $(5,6$, 10). Neither overexpression nor knockdown of FKBP38 affected the activity of WT and mTOR mutants (Figure 4, B and C). Of note, the role of FKBP38 as a negative regulator of MTORC1 remains highly debated $(81,82)$. DEPTOR is reported to bind to the FAT domain of mTOR and negatively regulate mTORC1 (4). Coimmunoprecipitation assays were performed to assess the interaction between individual mTOR mutants and DEPTOR. Notably, mTOR mutants in the F1 (L1460P, C1483F) but not F2 or F3 cluster coprecipitated less DEPTOR than WT mTOR (Figure 4D). As DEPTOR overexpression has been shown to inhibit WT mTORC1 activity (4), we examined the ability of DEPTOR overexpression to inhibit individual mTOR mutants. We showed that mTORC1 activities of L1460P (F1) and C1483F (F1) mutants were reduced when DEPTOR was overexpressed (Supplemental Figure 8), which suggests that DEPTOR may directly bind and thereby inhibit mTOR near the F1 cluster. Reduced binding to DEPTOR was also observed in 1 mTOR mutant in the K3 cluster (R2505P) (Figure 4D), which is consistent with other reports $(64,83)$. However, overexpression of DEPTOR did not inhibit the activity of the mTOR R2505P mutant (Supplemental Figure 8), implicating an indirect mechanism.

Subsets of mTOR-activating mutants are resistant to RAPTOR-mediated suppression. RAPTOR defines MTORC1 and presents substrates to mTOR, yet paradoxically negatively regulates mTORC1 upon overexpression or under low nutrient conditions (6). Accordingly, we examined the ability of RAPTOR overexpression to inhibit individual mTOR mutants, which led to the discovery of RAPTOR-sensitive and -insensitive mutants (Figure 5A). Similarly to WT mTOR, the mTORC1 activity of F1888L (F2), L2230V (K2), M2327I (K3), and R2505P (K3) mutants was reduced when RAPTOR was overexpressed. In contrast, the activity of L146OP (F1), C1483F (F1), T1977K (F3), and S2215F (K1) was not 
A Mutation cluster

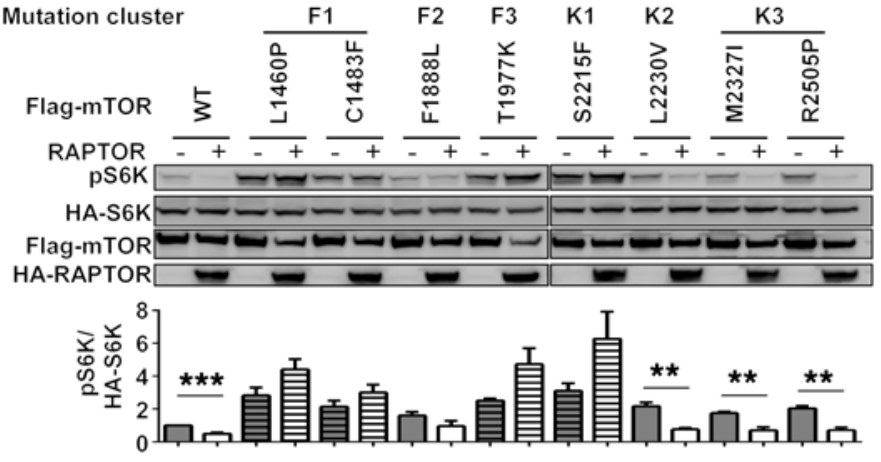

B

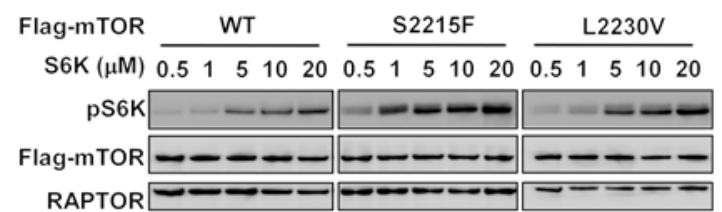

C

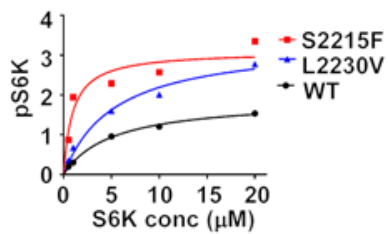

$\mathbf{E}$

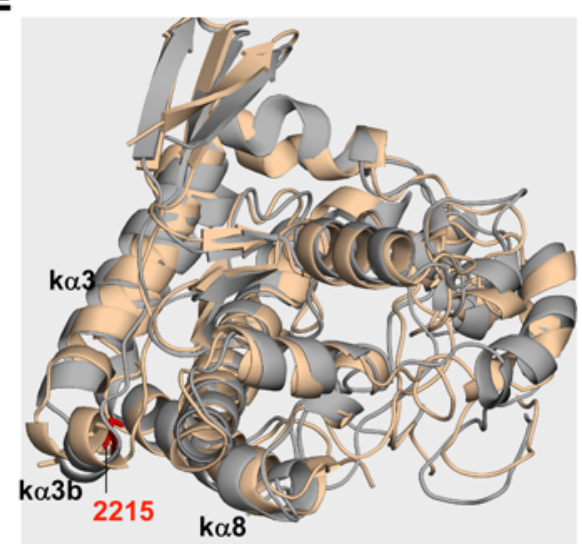

D
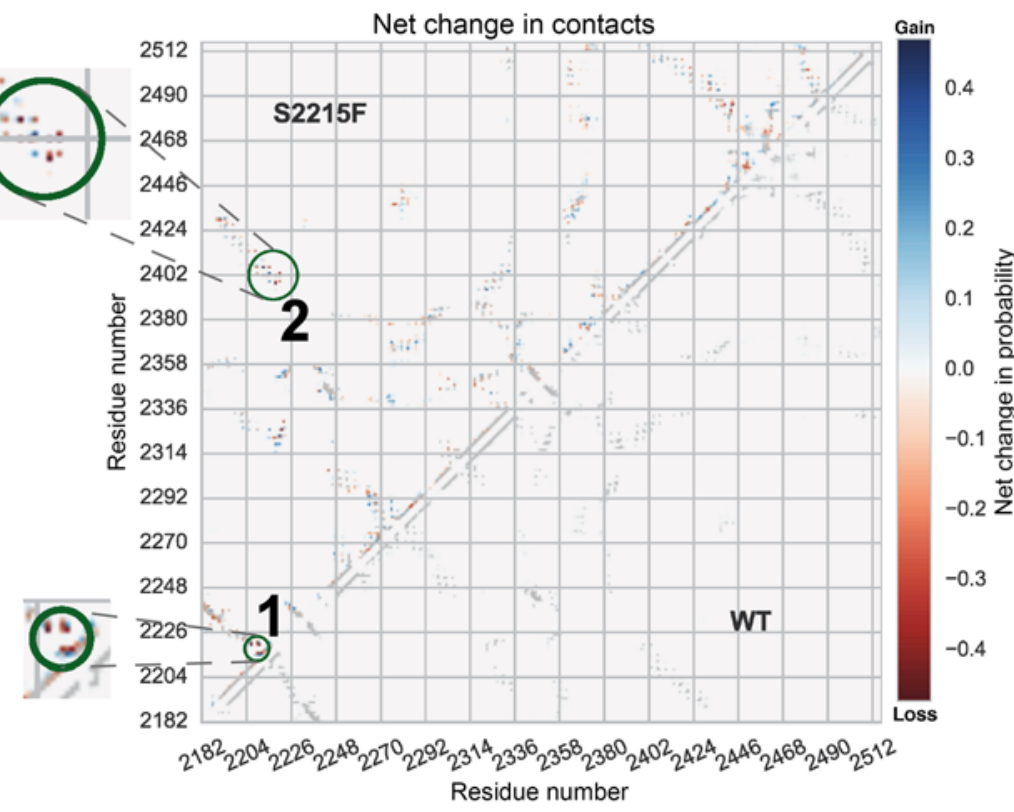

Residue number

Figure 5. Characterization of the response of mTOR-activating mutants to RAPTOR-mediated inhibition; mTOR kinase domain mutants exhibit increased kinase activity and display structural reorganization. (A) 293T cells, transfected with vectors expressing HA-S6K, indicated Flag-mTOR mutants, and either GFP or RAPTOR was subjected to immunoblot analysis using the indicated antibodies. Hatched bars indicate the mutants not inhibited by overexpressed RAPTOR. Densitometry of phosphorylated S6K versus HA-S6K from 3 independent experiments is shown (mean \pm SEM, $n=3$ independent experiments). ${ }^{*} P<0.01 ;{ }^{* *} P<0.001$ ( $t$ test). (B) In vitro kinase assays were carried out with the indicated Flag-tagged recombinant mTOR proteins in the presence of the indicated amount of recombinant HA-S6K. The recombinant Flag-mTOR and HA-S6K proteins were produced in $293 \mathrm{~T}$ cells by transient transfection of respective expression constructs followed by anti-Flag and anti-HA affinity purification, respectively. The reactions were analyzed by the indicated immunoblots. (C) Graph shows the quantitation of the phosphorylated S6K(T389) determined by densitometry versus S6K at different concentrations. (D) A contact map where each point $(x, y)$ gives the net change in probability of forming a contact between residue $x$ and residue $y$ between the mutant and WT, as estimated from simulations. The net contact change (blue indicating gain, red indicating loss) observed in the S2215F mutant is shown in the upper-left triangle, with WT shown in the bottom right to indicate the secondary structure present at corresponding locations. Disrupted contacts between residues 2214 and 2217 (green circle 1), residues 2218 and 2389 , and residues 2214 and 2402 (green circle 2) are highlighted. (E) Superposition of WT (gray) and S2215F (wheat) mTOR kinase domain structures at 501 ns of simulation, with the S2215F mutation shown in red sticks. Of note, k $\alpha 3 \mathrm{~b}$ is unwound and has moved further away from k $\alpha 8$ in $\mathrm{S} 2215 \mathrm{~F}$ simulation.

affected by RAPTOR overexpression. These data suggest that amino acid residues in the $\mathrm{F} 1, \mathrm{~F} 3$, and $\mathrm{K} 1$ clusters of mTOR might be important for sensing the negative inhibition exerted by RAPTOR. Interestingly, a recently solved mTORC1 structure consisting of mTOR and RAPTOR supports the interaction topology between mTOR and RAPTOR and features the roles of RAPTOR not only in substrate presentation but also in active site restriction (84).

mTOR kinase domain mutants exhibit increased kinase activity and display structural reorganization. Conceivably, the mutations in kinase domain may alter the conformation to affect the enzyme kinetics. Hence, we determined the enzyme kinetics of S2215F (K1) and L2230V (K2) mTOR mutants in phosphorylating S6K (Figure 5, $\mathrm{B}$ and C). Interestingly, S2215F increased $V_{\max }$ and decreased $\mathrm{Km}$ of mTORC1 kinase activity, whereas L2230V only affected $V_{\max }$ (Table 1). Since the crystal structure of C-terminal mTOR has been determined (80), we sought to examine the possible effects of the mTOR mutations on the conformation of C-terminal mTOR. Specifically, we performed 10 replicate molecular dynamics simulations for S2215F mutant; each trajectory was approximately 500 nanoseconds, and subsequent simulation data were analyzed for structural alternations indicative of rapid mutation-induced conformational changes. In a number of these simulations, we observed a displace- 
Table 1. Kinetic measurement of mTORC1 (WT or mutants) in in vitro kinase activity toward S6K

$\begin{array}{lccc} & \text { WT } & \text { S2215F } & \text { L2230V } \\ V_{\text {max }}(\mathrm{AU}) & 9.5 \pm 0.4 & 15.5 \pm 1.6 & 16.8 \pm 1.7 \\ K_{m(\text { (SбK) }}(\mu \mathrm{M}) & 5.1 \pm 0.6 & 0.9 \pm 0.4 & 5.3 \pm 1.5\end{array}$

ment of helix ka3b as contacts between residues 2214 and 2217 were disrupted (net fractional loss $14 \% \pm 6 \%$ in $\mathrm{S} 2215 \mathrm{~F}$ compared with WT; green circle 1) and also a decrease in interaction between helices $\mathrm{k} \alpha 3 \mathrm{~b}$ and $\mathrm{k} \alpha 8$ as contacts between residues 2218 and 2389 (net loss 63\% $\pm 6 \%$ ) and residues 2214 and 2402 (net loss 63\% \pm 7 $\%$ ) were disrupted (green circle 2) (Figure 5, D and E and Supplemental Figure 9). These simulation results could potentially explain the observed increase in kinase activity (85).

Kidney cancer-derived mTOR-activating mutants are resistant to REDD1-mediated inhibition and promote rapamycin-sensitive tumor formation in vivo. Clear cell kidney cancer is genetically noted for the biallelic inactivation of VHL tumor suppressor, which leads to the stabilization and accumulation of $\operatorname{HIFs}(37,50,54)$. DNA damage-inducible transcript 1 (REDD1) is a key transcriptional target of HIF1, and it negatively regulates mTORC1 (Supplemental Figure 10) (86-88). REDD1 activates TSC1/2 to inactivate RHEB and thereby inhibits mTORC1. On the other hand, active mTORC1 increases HIF1 protein levels through enhanced translation (89), which in turn induces REDD1, serving as a negative feedback loop (90). Based on this intricate interplay between HIF1 and mTORC1, we envisioned that mTOR-activating mutations might oppose REDD1-mediated inhibition and thereby abrogate the negative feedback loop linking HIF and mTOR. Indeed, overexpression of REDD1 was able to inhibit the WT mTOR, but not any of the tested activating mTOR mutants except F1888L (Figure $6 \mathrm{~A})$. The ability of activating mTOR mutations to escape negative regulation by REDD1 helps explain why mTOR-activating mutations occur frequently in clear cell kidney cancer in which REDD1 is upregulated by HIF in lieu of the VHL loss.

We next investigated whether these mTOR-activating mutations render tumor growth advantages in vivo. To assess potential phenotypic collaboration between VHL loss and mTORC1 hyperactivity, we depleted VHL using shRNA and overexpressed either WT or activating mutant mTOR in NIH/3T3 cells. As indicated by higher phosphorylated S6K, VHL-depleted NIH/3T3 cells expressing activating mTOR mutants displayed higher mTORC1 activity than those expressing WT mTOR (Figure 6B and Supplemental Figure 11). Furthermore, tumor allografts demonstrated that VHL-depleted NIH/3T3 cells expressing activating mTOR mutants (C1483F, S2215F, F1888L, T1977K, L2230V, and R2505P), but not nonactivating mutant (A1105P), grew significantly faster than those expressing WT mTOR (Figure 6, C and D and Supplemental Figures 12 and 13). To our knowledge, this is the first in vivo evidence demonstrating that mTOR-activating mutants directly contribute to tumor growth. Importantly, these tumor allografts were sensitive to rapamycin treatment (Figure 6, E and F).
It was reported that REDD1 activates TSC1/ 2 by sequestering 14-3-3 and thereby frees TSC1/2 to inactivate RHEB at the lysosome despite the inhibitory phosphorylation of TSC2 by upstream kinases at Ser939 $(74,88)$. Accordingly, we examined the TSC2 phosphorylation in VHL-depleted NIH/3T3 cells expressing WT or activating mutant mTOR (Supplemental Figure 14). Ser939 of TSC2 was strongly phosphorylated in VHL-depleted cells expressing either WT or mutant mTOR. Furthermore, overexpression of REDD1 was unable to suppress the tumor growth of VHL-depleted NIH/3T3 cells expressing activating mTOR C1483F mutant (Supplemental Figure 15). These results further support the notion that mTOR-activating mutations function downstream, bypass REDD1-TSC2-mediated inhibition, and thereby abrogate the negative feedback loop linking HIF and mTOR.

mTOR-activating mutants from different clusters display functional synergism. Collectively, we demonstrated 3 distinct mechanisms employed by specific mTOR mutation clusters to activate mTORC1, i.e., the loss of DEPTOR-dependent inhibition, the escape from RAPTOR suppression, and the increase of intrinsic kinase activity. All of these mechanisms could contribute to aberrant mTORC1 activity and thereby promote tumorigenesis. We hypothesized that functional complementation might occur between mechanistically distinct clusters, which might reveal not only the interdependence of individual activation mechanisms, but also the uncharacterized, potential mechanisms. To test this hypothesis, we systemically generated 28 mTOR mutants that carry 2 mutations from either the same or different clusters within a single mTOR molecule and determined their activity based on S6K phosphorylation (Figure 7, A and B). Remarkably, most double mutations across different clusters synergized strongly except for those involving the $\mathrm{K} 3$ cluster (Figure 7, A-C). Importantly, double mutations within the same clusters F1 (L1460P and C1483F) and K3 (M2327I and R2505P) did not yield synergism (Figure 7, A and B). Interestingly, F1888L (F2), a FAT domain mutation with no discernible features with respect to DEPTOR interaction and RAPTOR inhibition, synergized with all other mutations, thereby implicating yet-uncharacterized activating mechanisms. Overall, these data suggest that the geographically assigned mutation clusters coincide with the functional complementation groups.

mTOR mutants with 2 mutations from distinct clusters are hyperactive without RAG and RHEB. The strong synergism observed in mTOR double mutants prompted us to investigate their reliance on RAG and RHEB, their resistance to PRAS40, and their sensitivity to nutrient deprivation. Surprisingly, the examined mTOR double mutants were resistant to the overexpression of dominant negative RAGB ${ }^{\mathrm{GDP}} / \mathrm{RAGD}^{\mathrm{GTP}}$ (Figure $8 \mathrm{~A}$ and Supplemental Figure 16) and to the knockdown of RHEB (Figure 8B and Supplemental Figure 17), suggesting the loss of reliance on both RAG and RHEB for mTORC1 activation. On the other hand, these mutants were still inhibited by PRAS40 overexpression (Figure 8C and Supplemental Figure 18), indicating that synergistic double mutations did not alter substrate recognition through the TOR signaling (TOS) motif (91). Moreover, increased resistance of mTORC1 signaling to glucose, serum, or amino acid deprivation was observed in mTOR (C1483F/ T1977K, C1483F/S2215F, T1977K/S2215F, and F1888L/ L2230V) mutant HeLa cells (Figure 9, A-C and Supplemental 
A

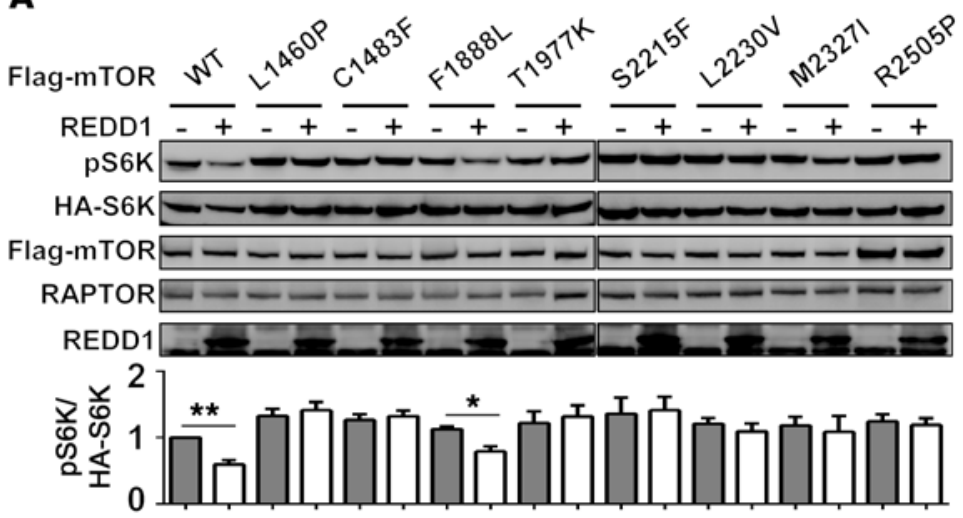

B

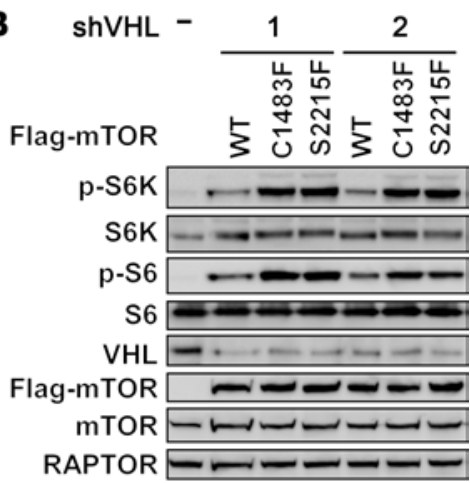

C

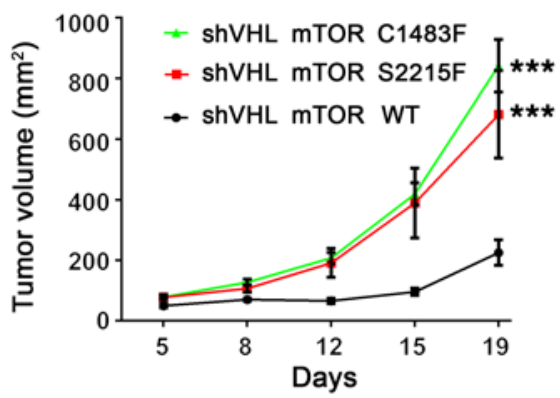

E

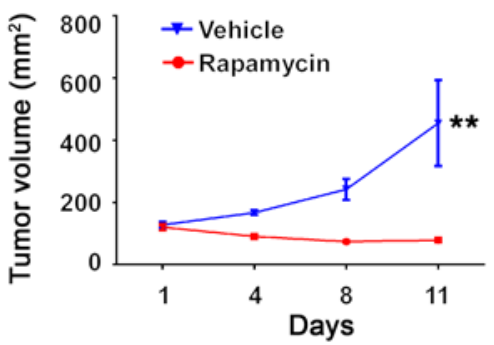

D

mTOR

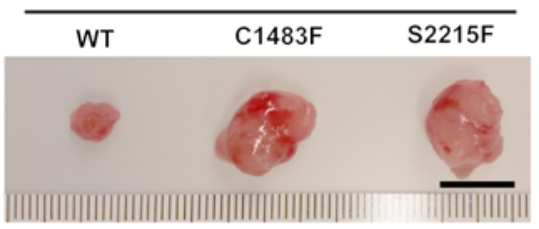

$\mathbf{F}$

ShVHL MTOR S2215F

Vehicle

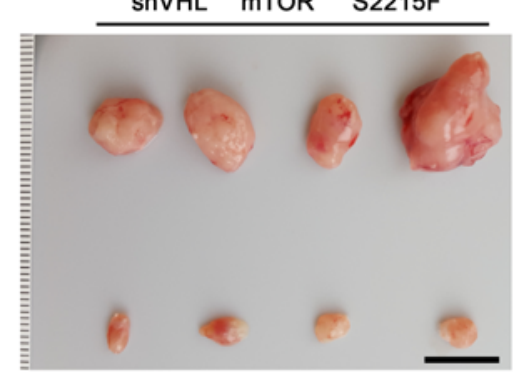

Figure 6. Kidney cancer-derived mTOR-activating mutants are resistant to REDD1-mediated inhibition and can promote rapamycin-sensitive tumor growth in vivo. (A) $293 \mathrm{~T}$ cells, transfected with vectors expressing HA-S6K, the indicated Flag-mTOR mutant, and either GFP or REDD1 were subjected to immunoblot analysis using the indicated antibodies. Densitometry of phosphorylated S6K versus HA-S6K from 3 independent experiments is shown (mean \pm SEM, $n=3$ independent experiments). ${ }^{*} P<0.05$; ${ }^{*} P<0.01$ ( $t$ test). (B) Expression of mTOR and shRNA against VHL in NIH/3T3 cells. NIH/3T3 cells stably expressing shRNA against luciferase or VHL (with 2 different constructs) as well as the indicated WT or mutant mTOR (C1483F and S2215F) were serum starved for 1 hour and restimulated with full media for 1 hour before being subjected to immunoblot analysis using the indicated antibodies. (C) NIH/3T3 cells expressing shVHL and the indicated WT or mutant mTOR (C1483F and S2215F) were implanted subcutaneously into the flanks of 6- to 8-week-old female NSG mice. Tumor size was measured for 19 days. Error bars represent SEM. ${ }^{* *} P<0.001$ ( 2 way ANOVA), $n=4$. (D) Image of harvested tumors used for $\mathbf{C}$ at day 19 . (E) NIH/3T3 cells expressing shVHL and mutant mTOR (S2215F) were implanted subcutaneously into the flanks of 6- to 8-week-old male NSG mice. When tumors reached 100 $\mathrm{mm}^{3}$, mice were randomized to received either vehicle or rapamycin treatment 3 times a week for an additional 11 days. Tumor size was measured for 11 days. Error bars represent SEM. ${ }^{* *} P<0.01$ ( 2 way ANOVA), $n=4$. (F) Image of harvested tumors used for $\mathbf{E}$ at day 11 after the start of the treatment.

Figure 19-21). Consequently, HeLa cells expressing mTOR double mutants were larger than HeLa cells expressing WT mTOR under full media or nutrient-deprived conditions (Figure 9D). Importantly, HeLa cells expressing mTOR double mutants displayed elevated resistance to rapamycin treatment compared with HeLa cells expressing WT mTOR or mTOR single mutants (Figure 9E and Figure 1D; Supplemental Figure 22).

\section{Discussion}

As a central player in the PI3K/AKT/mTOR pathway, mTOR functions as an integrator of intracellular and extracellular signals in eukaryotic cells to switch between anabolism and catabolism (2, $25,92)$. Accurate mTOR signaling is important for normal cellular homeostasis in metabolism, and dysregulation can result in diverse human diseases, including cancer, obesity, diabetes, and neurological disorders, as well as aging (1, 2, 93-95). Unlike mutations of the other PI3K/AKT/mTOR pathway components that have been extensively studied, the presence, the functional outcome, and the underlying activating mechanisms of mTOR missense mutations in contributing to human illness remain to be investigated. Recent mTORC1 inhibitor outlier studies in ccRCC, urothelial/bladder cancer, and thyroid cancer demonstrated that 
A

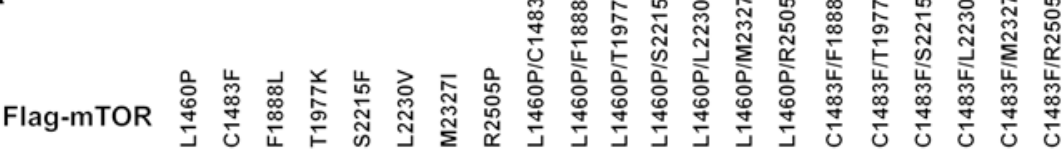

A-S6K

lag-mTOR

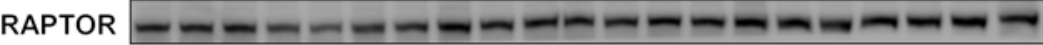

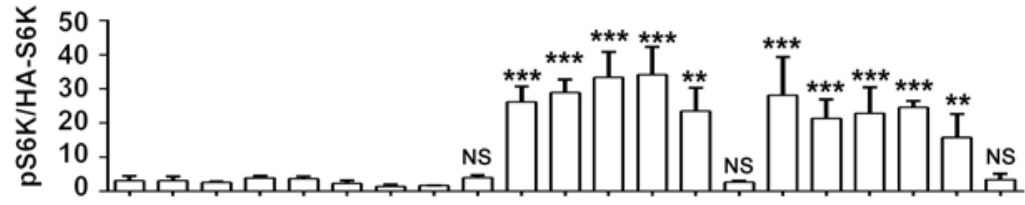

B
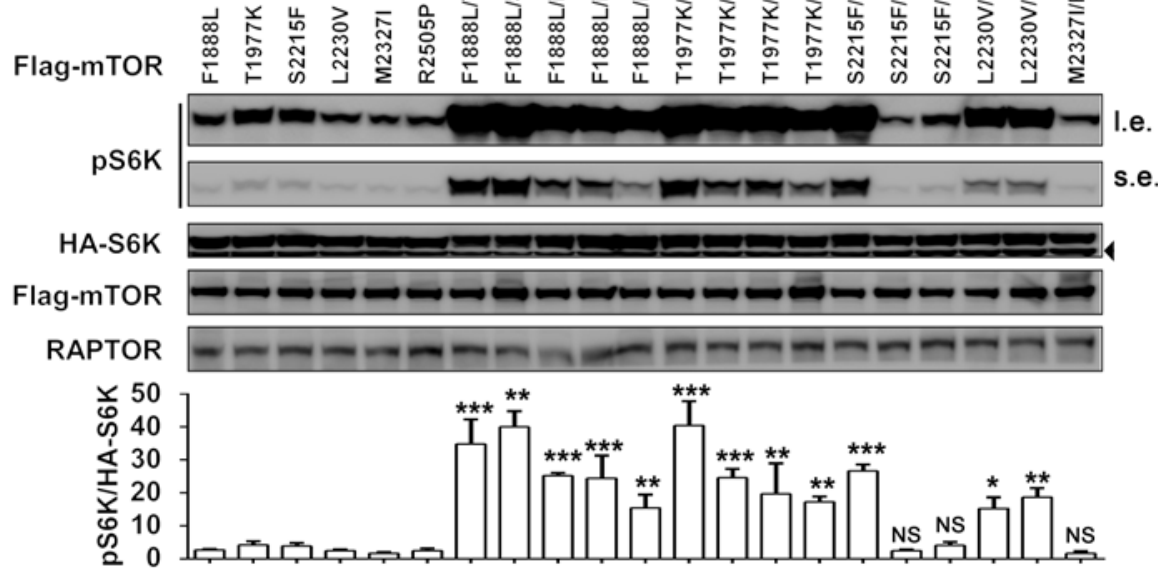

C

\begin{tabular}{|c|c|c|c|c|c|c|c|c|c|c|}
\hline $\begin{array}{l}\text { DEPTOR } \\
\text { RAPTOR }\end{array}$ & $\begin{array}{l}\text { DEPTOR } \\
\text { RAPTOR }\end{array}$ & & $\overline{-}$ & RAPTOR & $\begin{array}{l}\text { RAPTOR } \\
\text { Kinase }\end{array}$ & $\overline{\bar{z}}$ & $\overline{-}$ & $\begin{array}{c}\text { DEPTOR } \\
-\end{array}$ & \multicolumn{2}{|c|}{ Mechanism } \\
\hline F1 & F1 & & F2 & F3 & K1 & K2 & K3 & K3 & \multicolumn{2}{|l|}{ Cluster } \\
\hline L1460P & C1483F & & $1888 \mathrm{~L}$ & T1977K & S2215F & L2230V & M2327I & R2505P & \multicolumn{2}{|l|}{ Mutation } \\
\hline & \multirow[t]{2}{*}{ - } & & + & + & + & + & + & - & L1460P & F1 \\
\hline & & \multicolumn{2}{|r|}{+} & + & + & + & + & - & \begin{tabular}{|l|} 
C1483F \\
\end{tabular} & F1 \\
\hline & & & & + & + & + & + & + & F1888L & F2 \\
\hline & & & & & + & + & + & + & T1977K & $F 3$ \\
\hline & & & & & & + & - & - & S2215F & K1 \\
\hline & & - & \multicolumn{2}{|c|}{ No synergisn } & & & + & + & L2230V & $\mathrm{K} 2$ \\
\hline & & + & \multicolumn{2}{|c|}{ synergism } & & & & - & \begin{tabular}{|l|} 
M2327I \\
\end{tabular} & K3 \\
\hline & & & & & & & & & R2505P & K3 \\
\hline
\end{tabular}

Figure 7. Characterization of $m$ TOR double mutations. (A and B) 293T cells were transfected with vectors expressing HA-S6K and indicated Flag-mTOR mutants. Forty-eight hours later, cells were lysed and whole cell lysates were subjected to immunoblot analysis using the indicated antibodies. Densitometries of phosphorylated S6K (T389) versus HA-S6K are shown (mean \pm SEM, $n=3$ independent experiments). NS, $P>0.05$; ${ }^{*} P<0.05$; ${ }^{* *} P<0.01$; ${ }^{*} * P<0.001$ ( 2 way ANOVA test for synergism; ref. 109). I.e, long exposure; s.e, short exposure. Arrowheads denote cross-reactive bands. (C) Summary of functional complementation between 2 mTOR single mutations. Synergism is defined by $P<0.05$ in 2-way ANOVA test for synergism in panels $\mathbf{A}$ and $\mathbf{B}$. The activation mechanisms, including reduced binding to DEPTOR, resistance to RAPTOR overexpression-mediated inhibition, and altered kinase kinetics are indicated for each mutant.

HEAT domains are nonactivating mutations, which is consistent with previous reports $(64,83,96,97)$. Of note, mutations from different clusters could show different activities toward S6K in vitro, which is also reflected by the different tumor growth rates in vivo. These results further support that mutants of different clusters can be activated by different mechanisms. On the other hand, we found these mutations have little effect on mTORC2 activities. One study suggested mTOR mutations could increase mTORC2 activities, but the effects were very subtle (83), which is largely in line with our findings. Importantly, all activating mutations are sensitive to rapamycin treatment. Hence, cancer patients could be selected for mTOR inhibitor treatment based on mTOR mutations for basket trials (59). Interestingly, mTOR-activating mutations have recently been reported to associate with focal cortical dysplasia type II, which can lead to intractable epilepsy (98) that can be pharmacologically managed with rapamycin. This example and many yet to be discovered highlight roles

direct mTORC1 activation through either complete TSC1 loss or mTOR-activating mutation was associated with long-term therapeutic benefit (30-33). Furthermore, one recent study reported that mutations in $m$ TOR, TSC1, or TSC2 were more common in responders than nonresponders; however, a substantial fraction of responders had no mutations in the mTOR pathway (34).

Through systemic characterization of human kidney cancer-derived mTOR mutations, we demonstrated that mutations clustered at FAT and kinase domains are activating mutations for upregulating mTORC1 signals, while those dispersed at the of mTOR-activating mutations in human diseases beyond cancer and underscore the importance in characterizing and elucidating functional outcomes and the respective activating mechanisms of individual mTOR missense mutations.

Upstream signaling relays contributing to the eventual mTORC1 signaling output are complex $(1,19,99)$. Nevertheless, they mainly converge on 2 regulatory complexes of mTORC1, i.e. the Ragulator/RAG axis for amino acids and the TSC1/2/ RHEB axis for growth factor, receptor signaling, and other stress signals $(1,19)$. Interestingly, all of the mTOR-activating mutants 
A
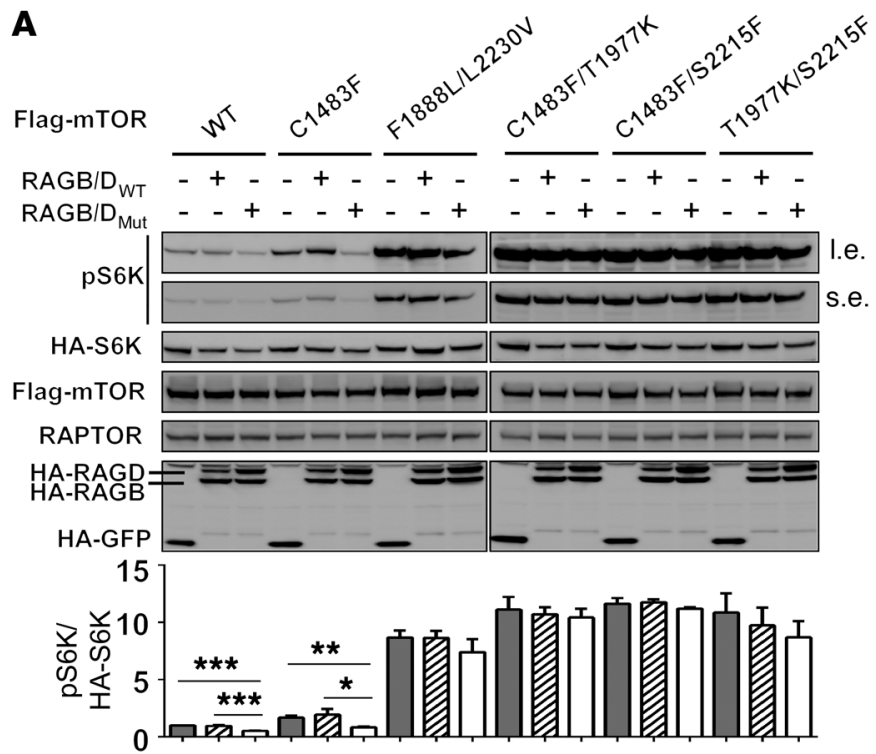

B

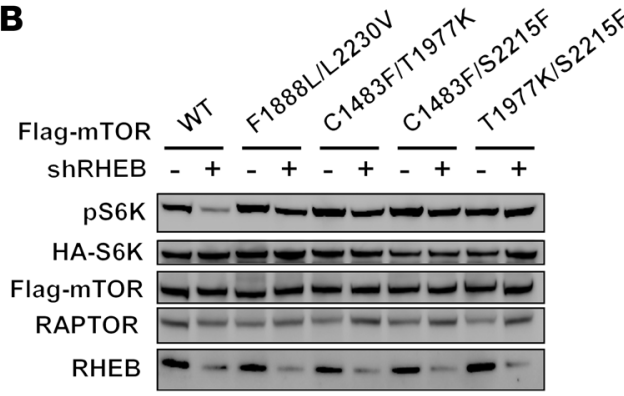

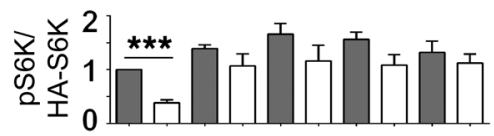

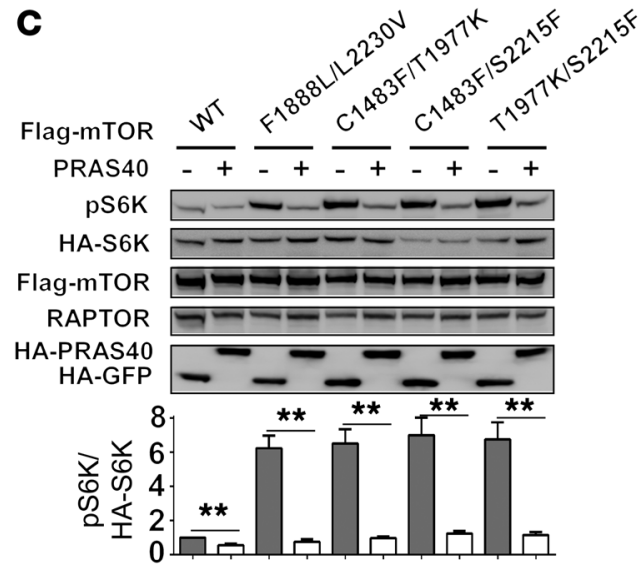

Figure 8. Characterization of the dependency of mTOR double mutants on RAG and RHEB. (A) 293T cells were transfected with vectors expressing HA-S6K, the indicated Flag-mTOR mutants, and either RAGB plus RAGD (RAGB/D ${ }_{W T}$ ) or dominant negative RAGB ${ }^{\text {GDP }}$ plus RAGD ${ }^{\text {GTP }}$ (RAGB/D ${ }_{\text {Mut }}$ ). Whole cell lysates were analyzed by the indicated immunoblots. (B) HeLa cells stably expressing shRNA against GFP or RHEB were transfected with vectors expressing HA-S6K and the indicated Flag-mTOR mutants and analyzed by the indicated immunoblots. (C) 293T cells were transfected with vectors expressing HA-S6K, the indicated Flag-mTOR mutants, and either GFP or PRAS40 and analyzed by the indicated immunoblots. For A-C, densitometry of phosphorylated S6K versus HA-S6K from 3 independent experiments is shown (mean \pm SEM, $n=3$ independent experiments). ${ }^{*} P<0.05 ;{ }^{* *} P<0.01$; ${ }^{* * *} P<0.001$ ( $t$ test).

examined were relatively resistant to serum or glucose deprivation, but similarly sensitive to amino acid deprivation when compared with WT mTOR. These data suggest that mTOR-activating mutations are more resistant to the TSC1/2/RHEB axis, while sensitive to the Ragulator/RAG axis. Consistent with this notion, mTOR-activating mutants are resistant to the inhibition mediated by overexpressed REDD1, which functions through activating TSC1/2 to inhibit mTORC1. Furthermore, mTOR-activating mutations confer tumor growth advantage to VHL-depleted cells in vivo. Based on TCGA pan-cancer studies (Supplemental Figure 1), most mTOR mutations in ccRCC are activating mutations that clustered in FAT and kinase domains, whereas mTOR mutations in several cancer types (e.g., uterus, lung) are in HEAT domain and likely represent passenger mutations. Our results offer an explanation for such observations, i.e., in ccRCC where $V H L$ loss stabilizes HIF that in turn upregulates REDD1 to negate mTORC1, mTOR-activating mutations could bypass this negative feedback and promote tumorigenesis (100). Importantly, tumors from NIH/3T3-expressing shVHL and mTOR-activating mutants were sensitive to rapamycin treatment in vivo, which is consistent with reported case studies on Rapalog long-term responders whose tumors carried mTOR-activating mutations $(30,31)$. Furthermore, the important collaboration between VHL loss (HIF activation) and mTORC1 activation presented by the current study in kidney cancer biology helps explain why kidney cancer was the first FDA-approved cancer type for rapalog-based therapies. Admittedly, it should be noted that activating of mTOR may be achieved through various means not limited to activating mutations in mTOR and the tumor heterogeneity may also complicate the usage of mTOR-activating mutations as biomarkers for predicting rapalog response (53). Nevertheless, our data indicate that when mTOR-activating mutation is an early "driver event" for tumorigenesis, it predicts the rapalogs response very well (101). Some mTOR-activating mutations characterized in this study were also detected in other cancer types (for example, $\mathrm{S} 2215 \mathrm{~F}$ was also identified in cervical squamous cell carcinoma, colorectal adenocarcinoma, and melanoma), and the role of these mTOR-activating mutations in the pathogenesis of other cancer types remains to be determined. However, as hypoxia is a common feature of most tumors $(102,103)$, mTORC1 activation may help tumor cells overcome the physiological brake on anabolism initiated by tissue hypoxia. A recent study also demonstrat- 
A

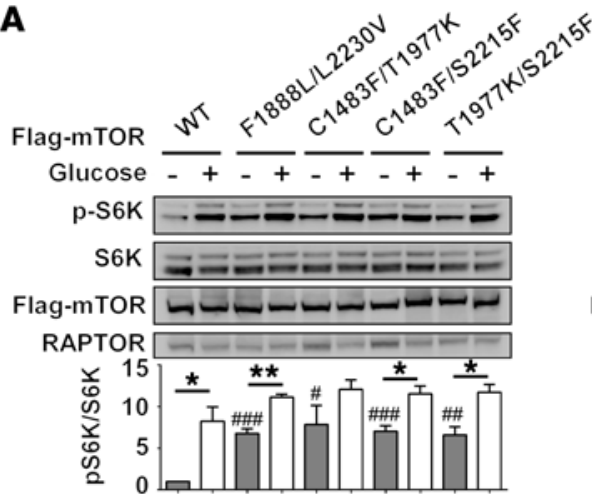

D

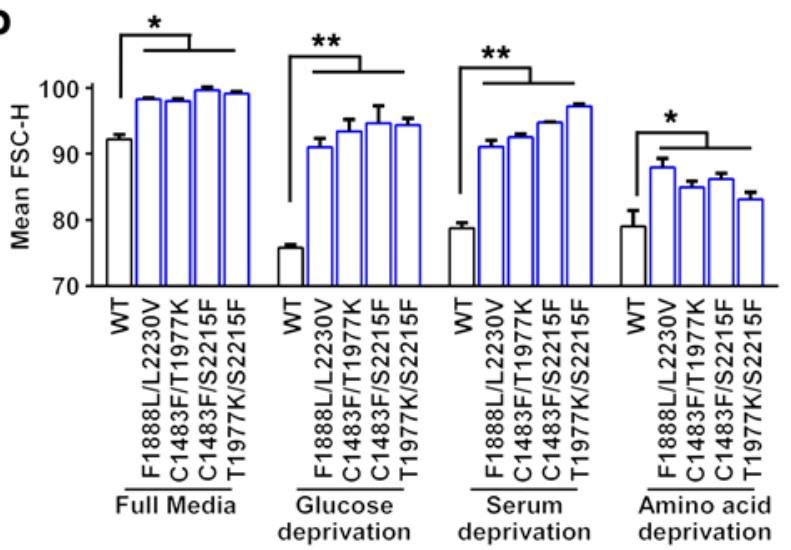

B

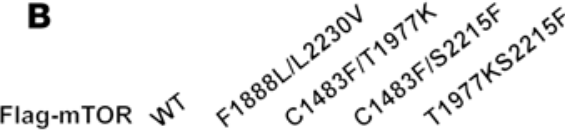

Serum $\frac{1}{-+}+\frac{1}{-+} \frac{\mathrm{d}}{-+} \frac{\mathrm{d}}{-+}$

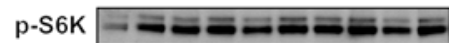

$\mathrm{S} 6 \mathrm{~K}$

Flag-mTOR ローーーーーーーーーー

RAPTOR

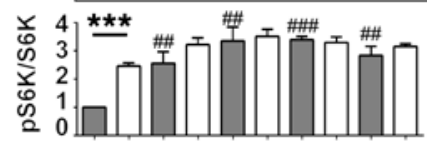

C

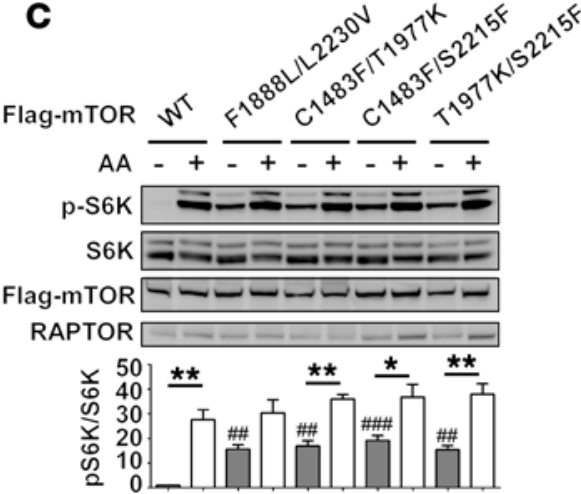

E

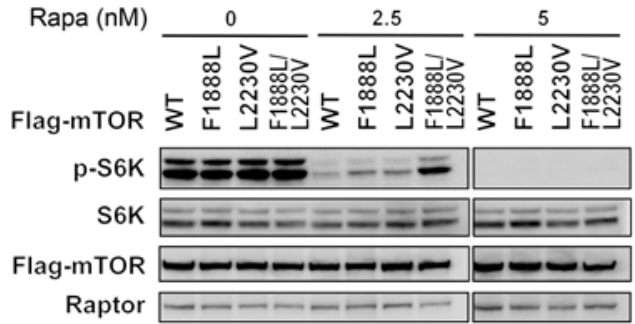

Figure 9. Characterization of nutrient dependence and sensitivity to rapamycin of mTOR double mutations. (A-C) HeLa cells expressing tetracyclineinducible WT or mutant mTOR were treated with doxycycline for 48 hours to induce mTOR. Cells were then starved for glucose (A), serum (B), or amino acids (C) for 1 hour or starved for 1 hour and restimulated with full media for 1 hour and subjected to immunoblot analysis. Densitometry of phosphorylated S6K versus HA-S6K from 3 independent experiments is shown (mean $\pm \mathrm{SEM}, n=3$ independent experiments). ${ }^{*} P<0.05$; ${ }^{* *} P<0.01$; ${ }^{* * *} P<0.001$ ( $t$ test). ${ }^{\#} P<0.05$; \#\# $P$ 0.01; \#\#P $<0.001$ ( $t$ test, comparison between indicated mutants and WT). (D) Tetracycline-inducible HeLa cells expressing WT or mutant mTOR under the indicated culture conditions were subjected to flow cytometry analysis for cell size. Data shown are the mean FSC-H from 3 independent experiments. Error bars represent SEM. ${ }^{*} P<0.05 ;{ }^{* *} P<0.01$ ( $t$ test). (E) Tetracycline-inducible HeLa cells expressing WT or mutant mTOR were treated with the indicated doses of rapamycin for 1 hour prior to immunoblot analysis using the indicated antibodies.

ed that mTOR-activating mutations could promote proliferation and transformation in vitro (97). Notably, the mTOR mutant (F1888L) was sensitive to REDD1 overexpression and yet promoted tumor growth when expressed in VHL-silenced NIH/3T3 cells, suggesting that this mutant may function through a unique mechanism. In fact, mTOR F1888L synergized with all other test mTOR mutants (Figure 7C).

mTORC1 consists of multiple subunits (mTOR, mLST8, RAPTOR, DEPTOR, and PRAS40) that are regulated by several interacting partners (RHEB, RAG, and FKBP38). While many studies have demonstrated the regulatory mechanisms of WT mTORC1 (2, 4-10, 19, 60-63), the activating mechanism of mTOR mutants is much less studied. One study assessed reduced binding between DEPTOR and mTOR as the only mechanism for all mTOR mutants (64). Here, we demonstrated that mTORactivating mutations can be divided into different clusters and each cluster is activated through at least 3 distinct mechanisms, including reduced binding to DEPTOR, increased resistance to RAPTOR overexpression-mediated inhibition, and enhanced intrinsic kinase activity. A summary of these features of selected activating mutations is listed in Supplemental Table 2. Based on our data, cluster F1 may define the region of mTOR interacting with DEPTOR and mutations (L1460P, C1483F) in this region could affect DEPTOR's direct binding to mitigate its negative regulation. Meanwhile, mutations of cluster F1 that map to TRD1 and the TRD1-proximal portion of TRD2 might destabilize interaction between TRD1 and the kinase domain. Cluster F3 maps to the HRD domain, and mutations of this cluster (T1977K) might also destabilize interaction between HRD and the kinase domain. Presumably, these kinds of destabilizations could lead to more openings for substrates to access the catalytic core. Mutations in the kinase domain (S2215F, L2230V) could increase the intrinsic catalytic rate $\left(V_{\max }\right)$, and the others $(\mathrm{S} 2215 \mathrm{~F})$ could increase the substrate access. These changes are likely to be caused by the conformational rearrangement induced by individual mutations. In another case, arginine-to-proline change of the mutation R2505P (cluster K3) could disrupt the $\alpha$-helix structure and distort the normal function of the negative regulatory loop nearby (104). Overall, all these results support the idea that individual mTOR mutation clusters can be activated by different mechanisms. Altogether, we exploited an approach based on the genetic complementation assay to examine the interplay among individual activating mech- 
anisms. Our results demonstrated that, in principle, 2 mutations from different mechanistic clusters cooperated, while 2 from the same did not. Furthermore, additional regulatory mechanisms beyond what we have presented might exist and warrant future investigation. Of note, mTOR belongs to the PI3K-related kinase (PIKKs) family, which has sequences similar to PI3K (105) and therefore may share the similar regulatory mechanisms with each other. Indeed, mutations in helical and kinase domains of the p110 subunit are activated by different mechanisms and can synergize when present in the same p110 molecule (106).

Intriguingly, cooperative double mTOR mutants became RAG and RHEB independent in mTORC1 activation. This is the only report, to our knowledge, showing this possibility. RHEB is the direct activator of mTORC1, but how this small GTPase activates mTOR remains elusive. Our study of RHEB-independent mTOR double mutants supports a potential mechanism by which RHEB activates mTORC1, i.e., RHEB functions to gauge upstream signaling strength and thereby gradually releases various restricted measures for adequate mTORC1 signaling output. Interestingly, mTOR double mutants display elevated resistance to low-dose rapamycin $(2.5 \mathrm{nM})$, but remain sensitive to high-dose rapamycin $(5-25 \mathrm{nM})$. It is tempting to speculate that, as limited drug perfusion of solid tumor could lead to subtherapeutic concentrations of rapalogs in poorly perfused tumor areas (107), tumor cells with single mTOR mutations could develop resistance to rapalog treatment by acquiring additional, mechanistically distinct mTOR mutations, which warrants further investigations. Our study and others on cancer-derived mTOR-activating mutations has not only deciphered a mechanistic blueprint concerning how mTORC1 is regulated, but also has laid the mechanistic basis for selecting patients whose diseases carry mTOR-activating mutations for the treatment of mTOR inhibitors.

\section{Methods}

Plasmid construction and shRNA- and siRNA-mediated knockdown. See Supplemental Methods for detailed information on plasmids, shRNA constructs, and siRNAs.

mTOR single mutations or double mutations were generated by introducing corresponding nucleotide changes into pcDNA3Flag-mTOR using the QuikChange II XL Site-Directed Mutagenesis Kit (Agilent). See Supplemental Table 3 for primers used for site-directed mutagenesis. All the constructs were confirmed by DNA sequencing. Lentiviral vectors carrying the indicated shRNA were cotransfected with pCMVAR8.2 and pHCMV.VSVG into 293T cells to generate lentivirus. Cells infected with lentivirus were under puromycin selection at $2 \mu \mathrm{g} / \mathrm{ml}$. siRNA oligonucleotides were reverse transfected using Lipofectamine RNAiMAX (Life Technologies) to a final concentration of $10 \mathrm{nM}$.

Cell culture, transfection, and immunoblot analysis. 293T (ATCC), HeLa (ATCC), and NIH/3T3 (ATCC) cells were cultured in DMEM (Invitrogen) supplemented with 10\% FBS, nonessential amino acids, L-glutamine, sodium pyruvate, and antibiotics (Invitrogen).

To assess mTORC1 signaling, 293T cells were seeded in 6-well plates at a density of $1.8 \times 10^{6}$ cells per well 24 hours before transfection and transfected with $1.5 \mu \mathrm{g}$ of vectors expressing WT or mutant mTOR and 50 ng of vectors expressing S6K, 4EBP1, or AKT1 using Lipofectamine 2000 according to the manufacturer's instructions
(Invitrogen). When indicated, cells were cotransfected with vectors expressing RAPTOR (3 $\mu \mathrm{g})$, DEPTOR $(3 \mu \mathrm{g})$, PRAS40 (1 $\mu \mathrm{g})$, RAGB (200 ng), RAGD (200 ng), RAGB ${ }^{\text {GDP }}$ (200 ng), RAGD ${ }^{\text {GTP }}$ (200 ng), or FKBP38 $(1 \mu \mathrm{g})$. At 48 hours after transfection, cells were harvested in ice-cold PBS buffer (137 mM NaCl, $2.7 \mathrm{mM} \mathrm{KCl}, 10 \mathrm{mM} \mathrm{Na}_{2} \mathrm{HPO}_{4}, 2$ $\mathrm{mM} \mathrm{KH} \mathrm{PO}_{4}$ ), pelleted, and lysed in RIPA buffer ( $150 \mathrm{mM} \mathrm{NaCl}, 1 \%$ NP-40, 1\% Na deoxycholate, 0.01 M sodium phosphate, $\mathrm{pH}$ 7.2, $0.1 \%$ SDS, $2 \mathrm{mM}$ EDTA, $50 \mathrm{mM} \mathrm{NaF}$ ) containing complete protease inhibitor (Roche) and phosphatase inhibitors (EMD/Millipore). Protein concentration was determined by the BCA Kit (Pierce). Equal amounts of proteins $(20-40 \mu \mathrm{g})$ were resolved by $10 \%$ or $4 \%-12 \%$ NuPAGE (Life Technologies) and transferred onto PVDF membrane (Immobilon-P, Millipore). Antibody detection was accomplished using the enhanced chemiluminescence method (Western Lightning, PerkinElmer) and the LAS-3000 Imaging System (Fujifilm). The immunoblot data were analyzed using ImageGauge software (Fujifilm).

See Supplemental Methods for detailed information on antibodies.

Generation of tetracycline-inducible HeLa and NIH/3T3 cell lines expressing WT or mutant mTOR. See Supplemental Methods for detailed information.

Nutrient deprivation and rapamycin treatment. For nutrient deprivation and refeeding, tet-inducible HeLa cell lines expressing WT or mutant mTOR were treated with doxycycline. Forty-eight hours later, cells were rinsed briefly with PBS, treated with complete DMEM media devoid of serum (for serum starvation), glucose (for glucose deprivation), or amino acid (for amino acid deprivation) for 1 hour, and restimulated with full complete media for 1 hour as indicated. Subsequently, cells were harvested and lysed as described above.

Rapamycin was obtained from Sigma-Aldrich (catalog R8781). Tet-inducible HeLa cell lines expressing WT or mutant mTOR were treated with the indicated concentrations of rapamycin or vehicle (DMSO) 48 hours after induction with doxycycline. Cells were harvested and lysed 1 hour later as described above.

Coimmunoprecipitation and in vitro kinase assays. See Supplemental Methods for detailed information.

Cell size measurement by flow cytometry. Cells were treated as indicated, harvested, and analyzed on a flow cytometer using the parameter mean forward scatter height (FSC-H), which is a measure of relative cell size. Flow cytometry was performed using a LSRFortessa (BD Biosciences), and data were analyzed using FACSDiva (BD Biosciences).

Molecular dynamics simulations. See Supplemental Methods for detailed information.

Mouse allograft study. For subcutaneous growth, 5 million $\mathrm{NIH} / 3 \mathrm{~T} 3$ cells expressing shRNA constructs and the tet-inducible mTOR were mixed 2:1 with Matrigel (BD Biosciences) and injected subcutaneously into 2 flanks of 6- to 8-week-old female NOD-SCID IL-2R $\gamma$ (NSG) mice (The Jackson Laboratory, stock no. 005557). Animals were administered with doxycycline $(2 \mathrm{mg} / \mathrm{ml}$; Sigma-Aldrich) plus sucrose $(50 \mathrm{mg} / \mathrm{ml})$ in their drinking water to induce $\mathrm{mTOR}$. The dimension of the tumors was measured by calipers, and tumor volume was calculated by the following formula: $1 / 2 \times$ width $^{2} \times$ length .

For drug treatment, 5 million NIH/3T3 cells expressing shRNA constructs and the tet-inducible mTOR were mixed 2:1 with Matrigel (BD Biosciences) and injected subcutaneously into 2 flanks of 6- to 8 -week-old male NSG mice. When tumor size reached $100 \mathrm{~mm}^{3}$, mice were randomized into 2 groups and were treated with either rapamy- 
cin $(8 \mathrm{mg} / \mathrm{kg})$ or vehicle $(0.25 \%$ PEG-400, 0.25\% Tween-80) 3 days per week by intraperitoneal injection as previously reported (108).

Statistics. Results are presented as mean \pm SEM. Except where otherwise specified, statistical significance was determined by unpaired 2-tailed Student's $t$ test. Except where otherwise specified, a $P$ value of less than 0.05 was considered significant.

Study approval. All mouse experiments were performed using a protocol approved by the IACUC at Memorial Sloan Kettering Cancer Center (protocol no. 10-07-012). All animal work was performed in accordance with NIH guidelines (Guide for the Care and Use of Laboratory Animals. National Academies Press. 2011.) and in compliance with all Memorial Sloan Kettering Cancer Center institutional requirements.

\section{Author contributions}

Design research studies were performed by JX, ZY, NR, EHC, and JJH. Conduct experiments were performed by JX, CGP, SKA, YD, TO, CHL, and DLP. Data acquisition was performed by JX, SKA, and DC. Data analysis was performed by JX, SKA, JDC, ZY, NR, EHC, and JJH. The manuscript was written by JX, EHC, and JJH. JX, ZY, $\mathrm{SH}, \mathrm{VRO}$, and JJH provided reagents. Study supervision was by JJH.

\section{Acknowledgments}

This work was supported by grants to JJH from the Functional Genomics Initiative and Cycle for Survival, as well as NIH NCI R01CA138505. This work was in part supported by the Cancer Center Support Grant from NCI to Memorial Sloan Ketterling (P30CA008748). JDC and DLP were partially supported by Sloan Kettering Institute. JX was supported by the Translational Research Oncology Training Fellowship from the Iris \& Junming Le Foundation. SKA was supported by the Louis V. Gerstner, Jr. Graduate School of Biomedical Sciences. We thank Smrutiben A. Mehta for technical assistance and Josh Fass for helpful software engineering suggestions. We also thank Jiangbin Ye, Wilhelm Palm, and Min Peng for helpful discussion and technical support. We thank Paul Jeng for editing the manuscript.

Address correspondence to: James J. Hsieh, Genitourinary Oncology Service, Human Oncology and Pathogenesis Program, Founding Director, MSK Translational Kidney Cancer Research Program, 415 E. 68th Street, ZRC762, New York, New York 10065, USA. Phone: 646.888.3263; E-mail: hsiehj@mskcc.org.
1. Zoncu R, Efeyan A, Sabatini DM. mTOR: from growth signal integration to cancer, diabetes and ageing. Nat Rev Mol Cell Biol. 2011;12(1):21-35.

2. Laplante M, Sabatini DM. mTOR signaling in growth control and disease. Cell. 2012;149(2):274-293.

3. Kim DH, et al. GbetaL, a positive regulator of the rapamycin-sensitive pathway required for the nutrient-sensitive interaction between raptor and mTOR. Mol Cell. 2003;11(4):895-904.

4. Peterson TR, et al. DEPTOR is an mTOR inhibitor frequently overexpressed in multiple myeloma cells and required for their survival. Cell. 2009;137(5):873-886

5. Hara K, et al. Raptor, a binding partner of target of rapamycin (TOR), mediates TOR action. Cell. 2002;110(2):177-189.

6. Kim DH, et al. mTOR interacts with raptor to form a nutrient-sensitive complex that signals to the cell growth machinery. Cell. 2002;110(2):163-175.

7. Sancak Y, et al. PRAS40 is an insulin-regulated inhibitor of the mTORC1 protein kinase. Mol Cell. 2007;25(6):903-915.

8. Vander Haar E, Lee SI, Bandhakavi S, Griffin TJ, Kim DH. Insulin signalling to mTOR mediated by the Akt/PKB substrate PRAS40. Nat Cell Biol. 2007;9(3):316-323.

9. Thedieck K, et al. PRAS40 and PRR5-like protein are new $\mathrm{mTOR}$ interactors that regulate apoptosis. PLOS ONE. 2007;2(11):e1217.

10. Wang L, Harris TE, Roth RA, Lawrence JC. PRAS40 regulates mTORC1 kinase activity by functioning as a direct inhibitor of substrate binding.J Biol Chem. 2007;282(27):20036-20044.

11. Jacinto E, et al. Mammalian TOR complex 2 controls the actin cytoskeleton and is rapamycin insensitive. Nat Cell Biol. 2004;6(11):1122-1128.

12. Sarbassov DD, et al. Rictor, a novel binding partner of mTOR, defines a rapamycin-insensitive and raptor-independent pathway that regulates the cytoskeleton. Curr Biol. 2004;14(14):1296-1302.
13. Frias MA, et al. $\mathrm{mSin} 1$ is necessary for $\mathrm{Akt} / \mathrm{PKB}$ phosphorylation, and its isoforms define three distinct mTORC2s. Curr Biol. 2006;16(18):1865-1870.

14. Jacinto E, et al. SIN1/MIP1 maintains rictor-mTOR complex integrity and regulates Akt phosphorylation and substrate specificity. Cell. 2006;127(1):125-137.

15. Pearce LR, Sommer EM, Sakamoto K, Wullschleger S, Alessi DR. Protor-1 is required for efficient mTORC2-mediated activation of SGK1 in the kidney. Biochem J. 2011;436(1):169-179.

16. Laplante M, Sabatini DM. An emerging role of mTOR in lipid biosynthesis. Curr Biol. 2009;19(22):R1046-R1052.

17. Ma XM, Blenis J. Molecular mechanisms of mTOR-mediated translational control. Nat Rev Mol Cell Biol. 2009;10(5):307-318.

18. Lamming DW, Sabatini DM. A Central role for mTOR in lipid homeostasis. Cell Metab. 2013;18(4):465-469.

19. Jewell JL, Guan KL. Nutrient signaling to mTOR and cell growth. Trends Biochem Sci. 2013;38(5):233-242.

20. Settembre C, et al. A lysosome-to-nucleus signalling mechanism senses and regulates the lysosome via $\mathrm{MTOR}$ and TFEB. EMBO J. 2012;31(5):1095-1108.

21. Yu L, et al. Termination of autophagy and reformation of lysosomes regulated by mTOR. Nature. 2010;465(7300):942-946.

22. Ganley IG, Lam du H, Wang J, Ding X, Chen S, Jiang X. ULK1.ATG13.FIP200 complex mediates mTOR signaling and is essential for autophagy. J Biol Chem. 2009;284(18):12297-12305.

23. Jung CH, et al. ULK-Atg13-FIP200 complexes mediate mTOR signaling to the autophagy machinery. Mol Biol Cell. 2009;20(7):1992-2003.

24. Hosokawa N, et al. Nutrient-dependent mTORC1 association with the ULK1-Atg13-FIP200 complex required for autophagy. Mol Biol Cell. 2009;20(7):1981-1991.

25. Kim YC, Guan KL. mTOR: a pharmacologic target for autophagy regulation. J Clin Invest. 2015;125(1):25-32.

26. Facchinetti V, et al. The mammalian target of rapamycin complex 2 controls folding and stability of Akt and protein kinase C. EMBO J. 2008;27(14):1932-1943.

27. Hudes G, et al. Temsirolimus, interferon alfa, or both for advanced renal-cell carcinoma. $N$ Engl Med. 2007;356(22):2271-2281.

28. Baselga J, et al. Everolimus in postmenopausal hormone-receptor-positive advanced breast cancer. N Engl JMed. 2012;366(6):520-529.

29. Motzer RJ, et al. Efficacy of everolimus in advanced renal cell carcinoma: a double-blind, randomised, placebo-controlled phase III trial. Lancet. 2008;372(9637):449-456.

30. Voss $\mathrm{MH}$, et al. Tumor genetic analyses of patients with metastatic renal cell carcinoma and extended benefit from mTOR inhibitor therapy. Clin Cancer Res. 2014;20(7):1955-1964.

31. Wagle N, et al. Activating mTOR mutations in a patient with an extraordinary response on a phase I trial of everolimus and pazopanib. Cancer Discov. 2014;4(5):546-553.

32. Iyer $\mathrm{G}$, et al. Genome sequencing identifies a basis for everolimus sensitivity. Science. 2012;338(6104):221.

33. Wagle N, et al. Response and acquired resistance to everolimus in anaplastic thyroid cancer. $N$ Engl JMed. 2014;371(15):1426-1433.

34. Kwiatkowski DJ, et al. Mutations in TSC1, TSC2, and MTOR Are Associated with Response to Rapalogs in Patients with Metastatic Renal Cell Carcinoma. Clin Cancer Res. 2016;22(10):2445-2452.

35. Ng CS, Wood CG, Silverman PM, Tannir NM, Tamboli P, Sandler CM. Renal cell carcinoma: diagnosis, staging, and surveillance. AJR Am J Roentgenol. 2008;191(4):1220-1232.

36. Valera VA, Merino MJ. Misdiagnosis of clear cell renal cell carcinoma. Nat Rev Urol. 2011;8(6):321-333.

37. Kaelin WG. The von Hippel-Lindau tumour sup- 
pressor protein: $\mathrm{O} 2$ sensing and cancer. Nat Rev Cancer. 2008;8(11):865-873.

38. Semenza GL. HIF-1 mediates metabolic responses to intratumoral hypoxia and oncogenic mutations. J Clin Invest. 2013;123(9):3664-3671.

39. Funakoshi T, Lee CH, Hsieh JJ. A systematic review of predictive and prognostic biomarkers for VEGF-targeted therapy in renal cell carcinoma. Cancer Treat Rev. 2014;40(4):533-547.

40. Escudier B, et al. Bevacizumab plus interferon alfa-2a for treatment of metastatic renal cell carcinoma: a randomised, double-blind phase III trial. Lancet. 2007;370(9605):2103-2111.

41. Rini BI, et al. Phase III trial of bevacizumab plus interferon alfa versus interferon alfa monotherapy in patients with metastatic renal cell carcinoma: final results of CALGB 90206. JClin Oncol. 2010;28(13):2137-2143.

42. Escudier B, et al. Sorafenib for treatment of renal cell carcinoma: Final efficacy and safety results of the phase III treatment approaches in renal cancer global evaluation trial. J Clin Oncol. 2009;27(20):3312-3318.

43. Escudier B, et al. Randomized phase II trial of firstline treatment with sorafenib versus interferon Alfa-2a in patients with metastatic renal cell carcinoma. JClin Oncol. 2009;27(8):1280-1289.

44. Motzer RJ, et al. Sunitinib in patients with metastatic renal cell carcinoma. JAMA. 2006;295(21):2516-2524.

45. Motzer RJ, et al. Sunitinib versus interferon alfa in metastatic renal-cell carcinoma. $N$ Engl J Med. 2007;356(2):115-124.

46. Sternberg CN, et al. Pazopanib in locally advanced or metastatic renal cell carcinoma: results of a randomized phase III trial. JClin Oncol. 2010;28(6):1061-1068.

47. Sternberg CN, et al. A randomised, double-blind phase III study of pazopanib in patients with advanced and/or metastatic renal cell carcinoma: final overall survival results and safety update. Eur J Cancer. 2013;49(6):1287-1296.

48. Rini BI, et al. Comparative effectiveness of axitinib versus sorafenib in advanced renal cell carcinoma (AXIS): a randomised phase 3 trial. Lancet. 2011;378(9807):1931-1939.

49. Hutson TE, et al. Axitinib versus sorafenib as firstline therapy in patients with metastatic renal-cell carcinoma: a randomised open-label phase 3 trial. Lancet Oncol. 2013;14(13):1287-1294.

50. Gossage L, Eisen T, Maher ER. VHL, the story of a tumour suppressor gene. Nat Rev Cancer. 2015;15(1):55-64.

51. Frew IJ, et al. pVHL and PTEN tumour suppressor proteins cooperatively suppress kidney cyst formation. EMBO J. 2008;27(12):1747-1757.

52 . Young AP, et al. VHL loss actuates a HIF-independent senescence programme mediated by $\mathrm{Rb}$ and p400. Nat Cell Biol. 2008;10(3):361-369.

53. Wei EY, Hsieh JJ. A river model to map convergent cancer evolution and guide therapy in RCC. Nat Rev Urol. 2015;12(12):706-712.

54. Cancer Genome Atlas Research Network. Comprehensive molecular characterization of clear cell renal cell carcinoma. Nature. 2013;499(7456):43-49.

55. Lin F, Zhang PL, Yang XJ, Prichard JW, Lun M, Brown RE. Morphoproteomic and molecular concomitants of an overexpressed and activated mTOR pathway in renal cell carcinomas. Ann Clin Lab Sci. 2006;36(3):283-293.

56. Pantuck AJ, et al. Prognostic relevance of the mTOR pathway in renal cell carcinoma: implications for molecular patient selection for targeted therapy. Cancer. 2007;109(11):2257-2267.

57. Abou Youssif T, et al. The mammalian target of rapamycin pathway is widely activated without PTEN deletion in renal cell carcinoma metastases. Cancer. 2011;117(2):290-300.

58. Voss MH, et al. Treatment outcome with mTOR inhibitors for metastatic renal cell carcinoma with nonclear and sarcomatoid histologies. Ann Oncol. 2014;25(3):663-668.

59. Sleijfer S, Bogaerts J, Siu LL. Designing transformative clinical trials in the cancer genome era. J Clin Oncol. 2013;31(15):1834-1841.

60. Kim E, Goraksha-Hicks P, Li L, Neufeld TP, Guan KL. Regulation of TORC1 by Rag GTPases in nutrient response. Nat Cell Biol. 2008;10(8):935-945.

61. Sancak Y, et al. The Rag GTPases bind raptor and mediate amino acid signaling to mTORC1. Science. 2008;320(5882):1496-1501.

62. Bai X, et al. Rheb activates mTOR by antagonizing its endogenous inhibitor, FKBP38. Science. 2007;318(5852):977-980.

63. Long X, Lin Y, Ortiz-Vega S, Yonezawa K, Avruch J. Rheb binds and regulates the mTOR kinase. Curr Biol. 2005;15(8):702-713.

64. Grabiner BC, et al. A diverse array of cancerassociated MTOR mutations are hyperactivating and can predict rapamycin sensitivity. Cancer Discov. 2014;4(5):554-563.

65. Motzer RJ, et al. Phase II randomized trial comparing sequential first-line everolimus and second-line sunitinib versus first-line sunitinib and second-line everolimus in patients with metastatic renal cell carcinoma. J Clin Oncol. 2014;32(25):2765-2772

66. Hsieh JJ, et al. Identification of efficacy biomarkers in a large metastatic renal cell carcinoma (mRCC) cohort through next generation sequencing (NGS): results from RECORD-3. JClin Oncol. 2015;33(15 suppl):4509.

67. Kang SA, et al. mTORC1 phosphorylation sites encode their sensitivity to starvation and rapamycin. Science. 2013;341(6144):1236566.

68. Kwiatkowski DJ, Manning BD. Molecular basis of giant cells in tuberous sclerosis complex. $N$ Engl $J$ Med. 2014;371(8):778-780.

69. Lee DF, et al. IKK beta suppression of TSC1 links inflammation and tumor angiogenesis via the mTOR pathway. Cell. 2007;130(3):440-455.

70. Inoki K, et al. TSC2 integrates Wnt and energy signals via a coordinated phosphorylation by AMPK and GSK3 to regulate cell growth. Cell. 2006;126(5):955-968.

71. Ma L, Chen Z, Erdjument-Bromage H, Tempst P, Pandolfi PP. Phosphorylation and functional inactivation of TSC2 by Erk implications for tuberous sclerosis and cancer pathogenesis. Cell. 2005;121(2):179-193.

72. Roux PP, Ballif BA, Anjum R, Gygi SP, Blenis J. Tumor-promoting phorbol esters and activated Ras inactivate the tuberous sclerosis tumor suppressor complex via p90 ribosomal S6 kinase. Proc Natl Acad Sci U S A. 2004;101(37):13489-13494.
73. Potter CJ, Pedraza LG, Xu T. Akt regulates growth by directly phosphorylating Tsc2. Nat Cell Biol. 2002;4(9):658-665.

74. Inoki K, Li Y, Zhu T, Wu J, Guan KL. TSC2 is phosphorylated and inhibited by Akt and suppresses mTOR signalling. Nat Cell Biol. 2002;4(9):648-657.

75. Manning BD, Tee AR, Logsdon MN, Blenis J, Cantley LC. Identification of the tuberous sclerosis complex-2 tumor suppressor gene product tuberin as a target of the phosphoinositide 3-kinase/akt pathway. Mol Cell.2002;10(1):151-162.

76. Betz C, Hall MN. Where is mTOR and what is it doing there?. JCell Biol. 2013;203(4):563-574.

77. Tee AR, Manning BD, Roux PP, Cantley LC, Blenis J. Tuberous sclerosis complex gene products, Tuberin and Hamartin, control mTOR signaling by acting as a GTPase-activating protein complex toward Rheb. Curr Biol. 2003;13(15):1259-1268.

78. Inoki K, Li Y, Xu T, Guan KL. Rheb GTPase is a direct target of TSC2 GAP activity and regulates mTOR signaling. Genes Dev. 2003;17(15):1829-1834.

79. Fingar DC, Salama S, Tsou C, Harlow E, Blenis J. Mammalian cell size is controlled by mTOR and its downstream targets S6K1 and 4EBP1/eIF4E. Genes Dev. 2002;16(12):1472-1487.

80. Yang H, Rudge DG, Koos JD, Vaidialingam B, Yang HJ, Pavletich NP. mTOR kinase structure, mechanism and regulation. Nature. 2013;497(7448):217-223.

81. Wang X, et al. Re-evaluating the roles of pro posed modulators of mammalian target of rapamycin complex 1 (mTORC1) signaling. J Biol Chem. 2008;283(45):30482-30492.

82. Uhlenbrock K, Weiwad M, Wetzker R, Fischer G, Wittinghofer A, Rubio I. Reassessment of the role of FKBP38 in the Rheb/mTORC1 pathway. FEBS Lett. 2009;583(6):965-970.

83. Ghosh AP, et al. Point mutations of the mTOR RHEB pathway in renal cell carcinoma. Oncotar get. 2015;6(20):17895-17910.

84. Aylett $\mathrm{CH}$, et al. Architecture of human mTOR complex 1. Science. 2016;351(6268):48-52.

85. Taylor SS, Kornev AP. Protein kinases: evolution of dynamic regulatory proteins. Trends Biochem Sci. 2011;36(2):65-77.

86. Brugarolas J, et al. Regulation of mTOR function in response to hypoxia by REDD1 and the TSC1/ TSC2 tumor suppressor complex. Genes Dev. 2004;18(23):2893-2904

87. Reiling JH, Hafen E. The hypoxia-induced paralogs Scylla and Charybdis inhibit growth by down-regulating S6K activity upstream of TSC in Drosophila. Genes Dev. 2004;18(23):2879-2892.

88. DeYoung MP, Horak P, Sofer A, Sgroi D, Ellisen LW. Hypoxia regulates TSC1/2-mTOR signaling and tumor suppression through REDD1-mediated 14-3-3 shuttling. Genes Dev. 2008;22(2):239-251.

89. Toschi A, Lee E, Gadir N, Ohh M, Foster DA. Differential dependence of hypoxia-inducible factors 1 alpha and 2 alpha on mTORC1 and mTORC2 J Biol Chem. 2008;283(50):34495-34499.

90. Kucejova B, et al. Interplay between pVHL and mTORC1 pathways in clear-cell renal cell carcinoma. Mol Cancer Res. 2011;9(9):1255-1265.

91. Schalm SS, Blenis J. Identification of a conserved motif required for mTOR signaling. Curr Biol. 2002;12(8):632-639. 
92. Shimobayashi M, Hall MN. Making new contacts: the mTOR network in metabolism and signalling crosstalk. Nat Rev Mol Cell Biol. 2014;15(3):155-162.

93. Dazert E, Hall MN. mTOR signaling in disease. Curr Opin Cell Biol. 2011;23(6):744-755.

94. Costa-Mattioli M, Monteggia LM. mTOR complexes in neurodevelopmental and neuropsychiatric disorders. Nat Neurosci. 2013;16(11):1537-1543.

95. Johnson SC, Rabinovitch PS, Kaeberlein M. mTOR is a key modulator of ageing and agerelated disease. Nature. 2013;493(7432):338-345.

96. Hardt M, Chantaravisoot N, Tamanoi F. Activating mutations of TOR (target of rapamycin). Genes Cells. 2011;16(2):141-151.

97. Yamaguchi $\mathrm{H}$, et al. Transforming somatic mutations of mammalian target of rapamycin kinase in human cancer. Cancer Sci. 2015;106(12):1687-1692.

98. Lim JS, et al. Brain somatic mutations in MTOR cause focal cortical dysplasia type II leading to intractable epilepsy. Nat Med.2015;21(4):395-400.

99. Hay N, Sonenberg N. Upstream and downstream of mTOR. Genes Dev. 2004;18(16):1926-1945.

100. Hakimi AA, Pham CG, Hsieh JJ. A clear picture of renal cell carcinoma. Nat Genet. 2013; 45(8):849-850.

101.Voss MH, Hsieh JJ. Therapeutic guide for mTOuRing through the Braided Kidney Cancer Genomic River. Clin Cancer Res. 2016;22(10):2320-2322.

102.Semenza GL. Oxygen sensing, hypoxia-inducible factors, and disease pathophysiology. Annu Rev Pathol. 2014;9:47-71.

103. Wilson WR, Hay MP. Targeting hypoxia in cancer therapy. Nat Rev Cancer. 2011;11(6):393-410.

104.Edinger AL, Thompson CB. An activated mTOR mutant supports growth factor-independent, nutrient-dependent cell survival. Oncogene. 2004;23(33):5654-5663.

105. Lempiäinen H, Halazonetis TD. Emerging com- mon themes in regulation of PIKKs and PI3Ks. ЕМВО J. 2009;28(20):3067-3073.

106.Zhao L, Vogt PK. Helical domain and kinase domain mutations in p110alpha of phosphatidylinositol 3-kinase induce gain of function by different mechanisms. Proc Natl Acad Sci U S A. 2008;105(7):2652-2657.

107. Tannock IF, Lee CM, Tunggal JK, Cowan DS, Egorin MJ. Limited penetration of anticancer drugs through tumor tissue: a potential cause of resistance of solid tumors to chemotherapy. Clin Cancer Res. 2002;8(3):878-884.

108. Woodrum C, Nobil A, Dabora SL. Comparison of three rapamycin dosing schedules in $\mathrm{A} / \mathrm{J} \mathrm{Tsc2+/-}$ mice and improved survival with angiogenesis inhibitor or asparaginase treatment in mice with subcutaneous tuberous sclerosis related tumors. J Transl Med. 2010;8:14.

109. Slinker BK. The statistics of synergism. J Mol Cell Cardiol.1998;30(4):723-731. 